\title{
JOBS: Joint-Sparse Optimization from Bootstrap Samples
}

\author{
Luoluo Liu, Student Member, IEEE, Sang (Peter) Chin, Member, IEEE, Trac D. Tran, Fellow, IEEE
}

\begin{abstract}
Classical signal recovery based on $\ell_{1}$ minimization solves the least squares problem with all available measurements via sparsity-promoting regularization. In practice, it is often the case that not all measurements are available or required for recovery. Measurements might be corrupted/missing or they arrive sequentially in streaming fashion. In this paper, we propose a global sparse recovery strategy based on subsets of measurements, named JOBS, in which multiple measurements vectors are generated from the original pool of measurements via bootstrapping, and then a joint-sparse constraint is enforced to ensure support consistency among multiple predictors. The final estimate is obtained by averaging over the $K$ predictors. The performance limits associated with different choices of number of bootstrap samples $L$ and number of estimates $K$ is analyzed theoretically. Simulation results validate some of the theoretical analysis, and show that the proposed method yields state-of-theart recovery performance, outperforming $\ell_{1}$ minimization and other existing bootstrap-based techniques in the challenging case of low levels of measurements. Our proposed framework is also preferable over other bagging-based methods in the streaming setting since it yields better recovery performances with small $K$ and $L$ for data-sets with large sizes.
\end{abstract}

\section{INTRODUCTION}

In Compressed Sensing (CS) and sparse recovery, solutions to the linear inverse problem in the form of least squares plus a sparsity-promoting penalty term have been intensively studied. Formally speaking, a the measurements vector $\boldsymbol{y} \in \mathbb{R}^{m}$ is generated by $\boldsymbol{y}=\boldsymbol{A} \boldsymbol{x}+\boldsymbol{z}$, where $\boldsymbol{A} \in \mathbb{R}^{m \times n}$ is the sensing matrix, $\boldsymbol{x} \in \mathbb{R}^{n}$ is the sparse coefficient with very few nonzero entries and $z$ is a bounded noise vector. The problem of interest is finding the sparse vector $\boldsymbol{x}$ given $\boldsymbol{A}$ as well as $\boldsymbol{y}$. However, directly minimizing the support size is proven to be NP-hard [1]. Instead, a convex regularizer is preferable. Among various choices, the $\ell_{1}$ norm is the most commonly used. The noiseless case is referred to as Basis Pursuit (BP):

$$
\mathbf{P}_{\mathbf{1}}: \min \|\boldsymbol{x}\|_{1} \text { s.t. } \boldsymbol{y}=\boldsymbol{A} \boldsymbol{x} .
$$

The noisy version is known as basis pursuit denoising [2], or least absolute shrinkage and selection operator (LASSO) [3]:

$$
\mathbf{P}_{\mathbf{1}}^{\boldsymbol{\epsilon}}: \min \|\boldsymbol{x}\|_{1} \text { s.t. }\|\boldsymbol{y}-\boldsymbol{A} \boldsymbol{x}\|_{2} \leq \epsilon .
$$

Luoluo Liu is with Department of Electrical Engineering, Johns Hopkins University, Baltimore, MD, 21210

Prof. S. Chin is with Department of Computer Science \& Hariri Institute of Computing, Boston University, Boston, MA, 02215 and Department of Electrical Engineering, Johns Hopkins University, Baltimore, MD, 21210

Prof. T. Tran is with Department of Electrical Engineering, Johns Hopkins University, Baltimore, MD, 21210

This work is partially supported by National Science Foundation under Grants xxx and Air Force Office of Scientific Research xxx, Manuscript revised Nov 16, 2018
The performance of $\ell_{1}$ minimization in recovering the true sparse solution has been thoroughly investigated in CS literature $[4]-[7]$. CS theory reveals that when the true solution is sparse and if the number of measurements is large enough, then the solution to (1) converges to the ground truth and (2) converges to its neighbourhood with high probability [4].

Unfortunately, in practice, all measurements may not be available. Some parts of the data can be missing or severely corrupted. In streaming settings, measurements might be available sequentially or in small batches. Wasting valuable time and buffering memory might not be the optimal strategy.

Alternatively, for sparse recovery or sparse-representationbased classification, many schemes use local observations and show promising performances $[8]-[11]$. It is not surprising since the number of measurements collected is usually much larger than lower bounds suggested by theory. However, proper choices of subset(s) differ between applications and require case-by-case treatment. Prior knowledge helps significantly in the selection process. For example, image data-sets may have large variance overall but relative invariance within local regions, choosing to work with image patches performs well in dictionary learning and deep learning [8], [12].

Without any prior information, a natural method is sampling uniformly at random with replacement, termed bootstrap [13]. It performs reasonably well when all measurements are equally good. In CS theory, some random matrices have been proven to be good sensing matrices. These operators act by shuffling and recombining entries of the original measurements. Consequently, any spatial or temporal structure would be destroyed, making the measurements even more democratic.

To incorporate the information from multiple estimates, the Bagging [14] procedure was proposed. It solves objectives multiple times independently from bootstrap samples and then averages over multiple predictions. Applying Bagging in sparse regression was shown to reduce estimation error when the sparsity level $s$ is high [14]. However, individually solved predictors aren't guaranteed to have the same support and in the worst case, their average can be quite dense: with its support size growing up to $K s$. To alleviate this problem, Bolasso was proposed [15], which firstly recovers the support of the final estimate by detecting the common support among $K$ individually solved predictors generated from bootstrap and then applies least squares on the common support. However, this strategy is very aggressive. When the noise level is high, it commonly recovers the zero solution.

In this paper, to resolve the support consistency issue in previous approaches and avoid issues caused by a twostep process, we propose to enforce row sparsity among all 
predictors using the $\ell_{1,2}$ norm within the iterative optimization loop. The entire process is as follows. First, we draw $L$ samples from $m$ measurements with replacement. Then we repeat this sampling process $K$ times to generate $\mathcal{I}_{1}, \mathcal{I}_{2}, \ldots, \mathcal{I}_{K}$, each of size $L$. This sampling process returns $K$ multi-sets of the original data $\left\{\boldsymbol{y}\left[\mathcal{I}_{1}\right], \boldsymbol{A}\left[\mathcal{I}_{1}\right]\right\},\left\{\boldsymbol{y}\left[\mathcal{I}_{2}\right], \boldsymbol{A}\left[\mathcal{I}_{2}\right]\right\} \ldots,\left\{\boldsymbol{y}\left[\mathcal{I}_{K}\right], \boldsymbol{A}\left[\mathcal{I}_{K}\right]\right\}$. Here we introduce the notation $(\cdot)[\mathcal{I}]$ : for a set (multi-set) $\mathcal{I}$, the operation $[\mathcal{I}]$ takes rows supported on $\mathcal{I}$ and throws away all other rows in the complement $\mathcal{I}^{c}$. For each solution $\boldsymbol{x}_{j}$ that corresponds to its data pair $\left\{\boldsymbol{y}\left[\mathcal{I}_{j}\right], \boldsymbol{A}\left[\mathcal{I}_{j}\right]\right\}$, we enforce the row sparsity constraint $\ell_{1,2}$ penalty on them to enforce the same support among all predictors. The $\ell_{1,2}$ norm penalty is defined as: $\|\boldsymbol{X}\|_{1,2}=\sum_{i}\left\|\boldsymbol{x}[i]^{\boldsymbol{T}}\right\|_{2}$, where $\boldsymbol{x}[i]$ denotes the $i$-th row of $\boldsymbol{X}$. The final estimates $\boldsymbol{x}^{\boldsymbol{J}}$ is obtained by averaging over all $K$ estimators. We coin the whole procedure JOBS (Joint-sparse Optimization from Bootstrap Samples). Other choices of row sparsity convex norms are suggested in [16]-[19].

The main contributions of this paper are: (i) We propose and demonstrate JOBS, employing the powerful bootstrapping, inspired from machine learning, and improves robustness of sparse recovery in noisy environments through the use of a collaborative recovery scheme. (ii) We explore the proposed strategy in-depth. Since the key parameters in our method is the bootstrap sample size $L$ and the number of bootstrap samples $K$, we derive various error bounds analytically with regards to these parameters. (iii) We also study optimal parameter settings and validate theory via extensive simulations.

For fair comparison to our method, we also extend and study Bagging and Bolasso, in the same setting. Solutions $\boldsymbol{x}_{1}^{\sharp}, \boldsymbol{x}_{2}^{\sharp}, \ldots \boldsymbol{x}_{K}^{\sharp}$ solved independently from the same observation as JOBS: $\left\{\boldsymbol{y}\left[\mathcal{I}_{1}\right], \boldsymbol{A}\left[\mathcal{I}_{1}\right]\right\},\left\{\boldsymbol{y}\left[\mathcal{I}_{2}\right], \boldsymbol{A}\left[\mathcal{I}_{2}\right]\right\} \ldots,\left\{\boldsymbol{y}\left[\mathcal{I}_{K}\right], \boldsymbol{A}\left[\mathcal{I}_{K}\right]\right\}$. Bagging takes average of multiple estimates and Bolasso conducts post-processing to ensure the support consistency of solution. Further contributions are: (iv) We explore the theoretical analysis for employing Bagging in sparse recovery. (v) Although the original Bagging and Bolasso use bootstrap ratio $L / m=1$, we studied the behavior of these two algorithms with multiple ratios $L / m$ from 0 to 1 , same as JOBS, to explore the optimal parameters as well as to make a fair comparison. (vi) We study a subsampling variation of the proposed scheme as an alternative to bootstrapping by simulations.

Simulation results show that our methods outperform all other methods when the number of measurements is small. While the number of measurements is large, acceptable performance of JOBS can be obtained with very small $L$ and $K$ and outperform Bagging and Bolasso, which potentially has an advantage in streaming settings in which $\ell_{1}$ minimization is not applicable and JOBS can achieve acceptable performance with small mini-batch sizes.

The outline of this paper is as follows: Section II illustrates the JOBS procedure; shows that it is a relaxation of $\ell_{1}$ minimization and provide some intuitions for analysis. Section III summarizes theoretical background material to analyze our algorithm. Section IV demonstrates all the major theoretical results of JOBS and Bagging with a generic $L / m$ ratio and $K$. Section $\mathrm{V}$ describes detailed analysis of the results in Section IV Finally, Section VI gives multiple simulation results comparing JOBS, Bagging, Bolasso, as well as $\ell_{1}$ minimization.

\section{Proposed Method: JOBS}

We first introduce a notation for the general form of the mixed $\ell_{p, q}$ norm of a matrix. The row sparsity penalty that we employed in our proposed method is a special case of this norm with $p=1, q=2$. The mixed $\ell_{p, q}$ norm on matrix $\boldsymbol{X}$ is defined as:

$$
\begin{aligned}
\|\boldsymbol{X}\|_{p, q} & =\left(\sum_{i=1}^{n}\left\|\boldsymbol{x}[i]^{\boldsymbol{T}}\right\|_{q}^{p}\right)^{1 / p} \\
& =\left\|\left(\left\|\boldsymbol{x}[1]^{\boldsymbol{T}}\right\|_{q},\left\|\boldsymbol{x}[2]^{\boldsymbol{T}}\right\|_{q}, \ldots,\left\|\boldsymbol{x}[n]^{\boldsymbol{T}}\right\|_{q}\right)^{\boldsymbol{T}}\right\|_{p},
\end{aligned}
$$

where $\boldsymbol{x}[i]$ denotes the $i$-th row of matrix $\boldsymbol{X}$. Intuitively, the mixed $\ell_{p, q}$ norm essentially takes $\ell_{q}$ norms on rows of $\boldsymbol{X}$ first; stacks those as a vector and then computes $\ell_{p}$ norm of this vector. Note when $p=q$, the $\ell_{p, p}$ norm of $\|\boldsymbol{X}\|$ is simply the $\ell_{p}$ vector norm on vectorized $\boldsymbol{X}$.

\section{A. JOBS}

Our proposed method JOBS can be accomplished in three steps. First, we generate bootstrap samples: The multiple bootstrap process generates $K$ multi-sets of the original data, which contains $K$ sensing matrices and measurements pairs: $\left\{\boldsymbol{y}\left[\mathcal{I}_{1}\right], \boldsymbol{A}\left[\mathcal{I}_{1}\right]\right\},\left\{\boldsymbol{y}\left[\mathcal{I}_{2}\right], \boldsymbol{A}\left[\mathcal{I}_{2}\right]\right\} \ldots,\left\{\boldsymbol{y}\left[\mathcal{I}_{K}\right], \boldsymbol{A}\left[\mathcal{I}_{K}\right]\right\}$. Second, we solve the collaborative recovery on those sets, the optimization in both noiseless and noisy forms. The noiseless case problem is: for all $j=1,2, \ldots, K$,

$$
\mathbf{J}_{\mathbf{1 2}}: \min \|\boldsymbol{X}\|_{1,2} \text { s.t. } \boldsymbol{y}\left[\mathcal{I}_{j}\right]=\boldsymbol{A}\left[\mathcal{I}_{j}\right] \boldsymbol{x}_{j},
$$

and the noisy counterpart can be expressed as: for some $\epsilon^{J}>0$,

$$
\mathbf{J}_{\mathbf{1 2}}^{\boldsymbol{\epsilon}^{J}}: \min \|\boldsymbol{X}\|_{1,2} \text { s.t. } \sum_{j=1}^{K}\left\|\boldsymbol{y}\left[\mathcal{I}_{j}\right]-\boldsymbol{A}\left[\mathcal{I}_{j}\right] \boldsymbol{x}_{j}\right\|_{2} \leq \epsilon^{J} .
$$

Proposed approaches in $\mathbf{J}_{\mathbf{1 2}}, \mathbf{J}_{\mathbf{1 2}}^{\epsilon^{J}}$ are in the form of Block(Group) sparse recovery [20] and numerous optimization methods can solve them such as [20]-[28].

Finally, the JOBS solution is obtained through averaging the columns of the solution of (4) or 5 : $X^{\sharp}$,

$$
\text { JOBS: } \quad \boldsymbol{x}^{\boldsymbol{J}}=\frac{1}{K} \sum_{j=1}^{K} \boldsymbol{x}_{j}^{\sharp} \text {. }
$$

All supports of $\boldsymbol{x}_{1}^{\sharp}, \boldsymbol{x}_{2}^{\sharp}, \ldots, \boldsymbol{x}_{K}^{\sharp}$ are the same because of the row sparsity constraint that we impose, and therefore the sparsity of the JOBS solution $x^{J}$ will not increase as in the Bagging case.

\section{B. Intuitive Explanation of why JOBS Works}

JOBS recovers the true sparse solution because it is a relaxation of the original $\ell_{1}$ minimization problem in multiple vectors. Let $x^{\star}$ be the true sparse solution; we will show that under some mild conditions, the row sparse minimization program recovers $\left(\boldsymbol{x}^{\star}, \boldsymbol{x}^{\star}, \ldots, \boldsymbol{x}^{\star}\right)$ correctly in Section IV. Thus the average over columns returns exactly the true solution $x^{\star}$.

We first demonstrate that JOBS is a two-step relaxation procedure of $\ell_{1}$ minimization. For a $\ell_{1}$ minimization as in equation (1) with a unique solution $\boldsymbol{x}^{\star}$, the Multiple Measurement Vectors (MMV) equivalence is: for $j=1,2, \ldots, K$

$$
\mathbf{P}_{\mathbf{1}}(K): \min \|\boldsymbol{X}\|_{1,1} \text { s.t. } \boldsymbol{y}=\boldsymbol{A} \boldsymbol{x}_{j},
$$


where $\|\boldsymbol{X}\|_{1,1}=\sum_{i}\left\|\boldsymbol{x}[i]^{\boldsymbol{T}}\right\|_{1}$. For the equivalence to the original problem, we have: if Single Vector Measurement (SMV) problem $\mathbf{P}_{\mathbf{1}}$ has a unique solution $\boldsymbol{x}^{\star}$, then the solution to the MMV problem $\mathbf{P}_{\mathbf{1}}(K)$ yields a row sparse solution $\boldsymbol{X}^{\star}=\left(\boldsymbol{x}^{\star}, \boldsymbol{x}^{\star}, \ldots, \boldsymbol{x}^{\star}\right)$. This result can be derived via contradiction. The reverse direction is also true: if the MMV problem $\mathbf{P}_{\mathbf{1}}(K)$ has a unique solution, it implies that the SMV problem $\mathbf{P}_{1}$ must also have a unique solution. One can refer to Lemma 20 and its proof in Appendix VIII-B for details.

Since the $\ell_{1,1}$ norm is separable for each elements of $\boldsymbol{X}$, it does not enforce support consistency. We therefore relax the $\ell_{1,1}$ norm in (7) to the $\ell_{1,2}$ norm. For all $j=1,2, . ., K$

$$
\mathbf{P}_{\mathbf{1 2}}(K): \min \|\boldsymbol{X}\|_{1,2} \text { s.t. } \boldsymbol{y}=\boldsymbol{A} \boldsymbol{x}_{j} .
$$

To obtain $\mathbf{J}_{\mathbf{1 2}}$ in (4), for each $\boldsymbol{x}_{j}$, we further relax the problem by dropping all constraints that are not in $\mathcal{I}_{j}$ from (8). This two-step relaxation process is illustrated in Figure 1

The noisy version can be analyzed similarly. We can formulate the MMV version of the original problem; relax the regularizer from $\ell_{1,1}$ norm to $\ell_{1,2}$ norm, and then further relax the objective function by dropping the constraints that are not on the selected subset $\mathcal{I}_{j}$ for $j$-th estimate $\boldsymbol{x}_{j}$ to obtain the proposed form $\mathbf{J}_{\mathbf{1 2}}^{\epsilon^{J}}$.

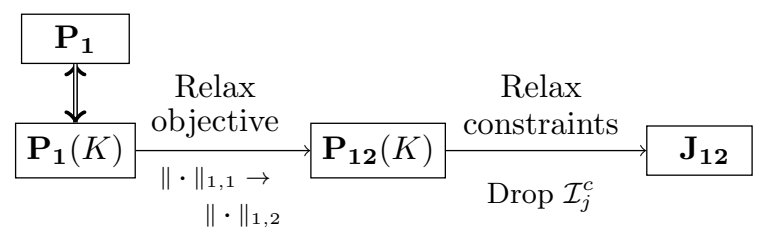

Fig. 1. Flowchart explaining JOBS as a relaxation of $\ell_{1}$ minimization

\section{PRELIMINARIES}

We summarize the theoretical results that are needed for understanding and analyzing our algorithm mathematically. We are going to introduce block sparsity, Null Space Property (NSP), as well as Restricted Isometry Property (RIP).

\section{A. Block (non-overlapping group) Sparsity}

Because row sparsity is a special case of block sparsity (or non-overlapping group sparsity), we therefore can employ the tools from block sparsity to analyze our problem. To start, recall the definition of block sparsity as in [17]:

Definition 1 (Block Sparsity). $\boldsymbol{x} \in \mathbb{R}^{n}$ is $s$-block sparse with respect to a partition $\mathcal{B}=\left\{\mathcal{B}_{1}, \mathcal{B}_{2}, \ldots, \mathcal{B}_{K}\right\}$ of $\{1,2, \ldots, n\}$ if $\boldsymbol{x}=\left(\boldsymbol{x}\left[\mathcal{B}_{1}\right], \ldots \boldsymbol{x}\left[\mathcal{B}_{K}\right]\right)$, the norm $\|\boldsymbol{x}\|_{2,0 \mid \mathcal{B}}:=$ $\sum_{i=1}^{K} \mathbb{1}\left\{\left\|\boldsymbol{x}\left[\mathcal{B}_{i}\right]\right\|_{2}>0\right\} \leq s$ and the relaxation $\ell_{1,2}$ norm $\|\boldsymbol{x}\|_{2,1 \mid \mathcal{B}}:=\sum_{i=1}^{K}\left\|\boldsymbol{x}\left[\mathcal{B}_{i}\right]\right\|_{2}$.

The block sparsity level $\|\boldsymbol{x}\|_{2,0 \mid \mathcal{B}}$ counts the number of nonzero blocks of the given block partition $\mathcal{B}$. Block sparsity is also a generalization of standard sparsity. Usually, for the same sparse vector $\boldsymbol{x}$, the group sparsity level is smaller than the sparsity level. Therefore knowing the group sparse information may reduce the number of minimum measurements needed comparing to standard sparse recovery.
We can see that $\ell_{1,2}$ minimization is a special case of block sparse minimization [17], with each element in the group partition containing the indices of each row. Therefore, the analysis of our algorithm follows similar analyses in the studies of block sparsity such as Block Null Space Property (BNSP) [29], Block Restricted Isometry Property (BRIP) [30].

\section{B. Null Space Property (NSP) and Block-NSP (BNSP)}

The NSP [31] for standard sparse recovery and block sparse signal recovery are given in the two following theorems.

Theorem 2 (NSP). Every $s$-sparse signal $\boldsymbol{x} \in \mathbb{R}^{n}$ is a unique solution to $\mathbf{P}_{\mathbf{1}}$ : $\min \|\boldsymbol{x}\|_{1}$ s.t. $\boldsymbol{y}=\boldsymbol{A} \boldsymbol{x}$ if and only if $\boldsymbol{A}$ satisfies NSP of order s. Namely, if for all $\boldsymbol{v} \in \operatorname{Null}(\boldsymbol{A}) \backslash\{\mathbf{0}\}$, such that for any set $\mathcal{S}$ of cardinality less than equals to $s$ $: \mathcal{S} \subset\{1,2, \ldots, n\}, \operatorname{card}(\mathcal{S}) \leq s$, the following is satisfied:

$$
\|\boldsymbol{v}[\mathcal{S}]\|_{1}<\left\|\boldsymbol{v}\left[\mathcal{S}^{c}\right]\right\|_{1},
$$

where $\boldsymbol{v}[\mathcal{S}]$ only has the vector values on a index set $\mathcal{S}$ and zero elsewhere.

Theorem 3 (BNSP). Every $s$-block sparse signal $\boldsymbol{x}$ respect to block assignment $\mathcal{B}, \boldsymbol{A}$ is unique solution to $\min \|\boldsymbol{x}\|_{1,2 \mid \mathcal{B}}$ s.t. $\boldsymbol{y}=\boldsymbol{A} \boldsymbol{x}$ if and only if $\boldsymbol{A}$ satisfies Block Null Space Property (BNSP) of order s:

For any set $\mathcal{S} \subset\{1,2, \ldots, n\}$ with $\operatorname{card}(\mathcal{S}) \leq s$, a matrix $\boldsymbol{A}$ is said to satisfy block null space property over $\mathcal{B}$ of order $s$, if

$$
\|\boldsymbol{v}[\mathcal{S}]\|_{1,2 \mid \mathcal{B}}<\left\|\boldsymbol{v}\left[\mathcal{S}^{c}\right]\right\|_{1,2 \mid \mathcal{B}},
$$

for all $\boldsymbol{v} \in \operatorname{Null}(\boldsymbol{A}) \backslash\{\mathbf{0}\}$, where $\boldsymbol{v}_{\mathcal{S}}$ denotes the vector equal to $\boldsymbol{v}$ on a block index set $\mathcal{S}$ and zero elsewhere.

\section{Restricted Isometry Property (RIP) and Block-RIP (BRIP)}

Although NSP directly characterizes the ability of success for sparse recovery, checking the NSP condition is computationally intractable, and it is also not suitable to use NSP for quantifying performance in noisy conditions since it is a binary (True or False) metric instead of in a continuous range. Restricted isometry property (RIP) is introduced for those purposes and there are many sufficient conditions based on RIP. Let us recall RIP [4] for standard sparse recovery and BRIP [30] for block sparse recovery.

Definition 4 (RIP). A matrix $\boldsymbol{A}$ with $\ell_{2}$-normalized columns satisfies RIP of order $s$ if there exists a constant $\delta_{s}(\boldsymbol{A}) \in[0,1)$ such that for every $s$-sparse $\boldsymbol{v} \in \mathbb{R}^{n}$, we have:

$$
\left(1-\delta_{s}(\boldsymbol{A})\right)\|\boldsymbol{v}\|_{2}^{2} \leq\|\boldsymbol{A} \boldsymbol{v}\|_{2}^{2} \leq\left(1+\delta_{s}(\boldsymbol{A})\right)\|\boldsymbol{v}\|_{2}^{2} .
$$

More generally, the RIP condition for block sparsity definitions (Definition 2 in [30]) are as the following:

Definition 5 (BRIP). A matrix $\boldsymbol{A}$ with $\ell_{2}$-normalized columns satisfies Block RIP with respect to block partition $\mathcal{B}$ of order $s$ if there exists a constant $\delta_{s \mid \mathcal{B}}(\boldsymbol{A}) \in[0,1)$ such that for every $s$-block sparse $\boldsymbol{v} \in \mathbb{R}^{n}$ over $\mathcal{B}$, we have:

$$
\left(1-\delta_{s \mid \mathcal{B}}(\boldsymbol{A})\right)\|\boldsymbol{v}\|_{2}^{2} \leq\|\boldsymbol{A} \boldsymbol{v}\|_{2}^{2} \leq\left(1+\delta_{s \mid \mathcal{B}}(\boldsymbol{A})\right)\|\boldsymbol{v}\|_{2}^{2}
$$

Again, if we take every entry as a block, the block sparsity RIP reduces to the standard RIP condition. 


\section{Noisy Recovery bounds based on RIP constants}

It is known that RIP conditions imply NSP conditions satisfied for both block sparse recovery and sparse recovery. More specifically, if the RIP constant in the order $2 s$ is strictly less than $\sqrt{2}-1$, then it implies that NSP is satisfied in the order of $s$. This applies to both classic $\ell_{1}$ sparse recovery and block sparse recovery.

The noisy recovery performance bound based on RIP constant for $\ell_{1}$ minimization problem and the noisy recovery bound for block sparse recovery based on BRIP constant are shown in the following two theorems.

Theorem 6 (Noisy recovery for $\ell_{1}$ minimization, Theorem 1.2 in [4]). Let $\boldsymbol{y}=\boldsymbol{A} \boldsymbol{x}^{\star}+\boldsymbol{z},\|\boldsymbol{z}\|_{2} \leq \epsilon, \boldsymbol{x}_{0}$ is s-sparse that minimizes $\left\|\boldsymbol{x}-\boldsymbol{x}^{\star}\right\|$ over all $s-$ sparse signals. If $\delta_{2 s}(\boldsymbol{A})<$ $\sqrt{2}-1, x^{\ell_{1}}$ be the solution of $\ell_{1}$ minimization, then

$$
\left\|\boldsymbol{x}^{\ell_{1}}-\boldsymbol{x}^{\star}\right\|_{2} \leq \mathcal{C}_{0}\left(\delta_{2 s}(\boldsymbol{A})\right) s^{-1 / 2}\left\|\boldsymbol{x}_{0}-\boldsymbol{x}^{\star}\right\|_{1}+\mathcal{C}_{1}\left(\delta_{2 s}(\boldsymbol{A})\right) \epsilon,
$$

where $\mathcal{C}_{0}(\cdot), \mathcal{C}_{1}(\cdot)$ are some constants, which are determined by RIP constant $\delta_{2 s}$. The form of these two constants terms are $\mathcal{C}_{0}(\delta)=\frac{2(1-(1-\sqrt{2}) \delta)}{1-(1+\sqrt{2}) \delta}$ and $\mathcal{C}_{1}(\delta)=\frac{4 \sqrt{1+\delta}}{1-(1+\sqrt{2}) \delta}$.

Theorem 7 (Noisy recovery for block sparse recovery, Theorem 2 in [17]). Let $\boldsymbol{y}=\boldsymbol{A} \boldsymbol{x}^{\star}+\boldsymbol{z},\|\boldsymbol{z}\|_{2} \leq \epsilon, \boldsymbol{x}_{0}$ is $s$-block sparse that minimizes $\left\|\boldsymbol{x}-\boldsymbol{x}^{\star}\right\|$ over all $s$-block sparse signals. If $\delta_{2 s \mid \mathcal{B}}(\boldsymbol{A})<\sqrt{2}-1, \boldsymbol{x}^{\ell_{1,2 \mid \mathcal{B}}}$ be the solution of block sparse minimization, then

$$
\begin{aligned}
\left\|\boldsymbol{x}^{\ell_{1,2 \mid \mathcal{B}}}-\boldsymbol{x}^{\star}\right\|_{2} & \leq \mathcal{C}_{0}\left(\delta_{2 s \mid \mathcal{B}}(\boldsymbol{A})\right) s^{-1 / 2}\left\|\boldsymbol{x}_{0}-\boldsymbol{x}^{\star}\right\|_{1,2 \mid \mathcal{B}} \\
& +\mathcal{C}_{1}\left(\delta_{2 s \mid \mathcal{B}}(\boldsymbol{A})\right) \epsilon
\end{aligned}
$$

where $\mathcal{C}_{0}(\cdot), \mathcal{C}_{1}(\cdot)$ are the same functions as in Theorem 6

\section{E. Sufficient Condition: Sample Complexity for Gaussian and Bernoulli Random Matrices}

Since checking either NSP or RIP conditions is computationally hard and it doesn't provide direct guidance for designing sensing matrices, some previous work built a relationship between sample complexity for random matrices to a designed RIP constant. The classical one is Theorem 5.2 in [32]:

Theorem 8 (Sufficient Condition: Sample Complexity). Let entries of $\boldsymbol{A} \in \mathbb{R}^{m \times n}$ from $\mathcal{N}(0,1 / m), 1 / \sqrt{m} \operatorname{Bern}(0.5)$. Let $\mu, \delta \in(0,1)$ and assume $m \geq \beta \delta^{-2}\left(s \ln (n / s)+\ln \left(\mu^{-1}\right)\right)$ for a universal constant $\beta>0$, then $\mathbb{P}\left(\delta_{s}(\boldsymbol{A}) \leq \delta\right) \geq 1-\mu$.

By rearranging the terms in this theorem, the sample complexity can be derived: when $m$ is in the order of $\mathcal{O}(2 s \ln (n / 2 s))$ and sufficient large, there is a high probability that the RIP constant of order $2 s$ is sufficiently small.

\section{TheORETICAL RESUlts FOR JOBS}

\section{A. BNSP}

Similarly to previous CS analysis in [4], we give the null space property to characterize the exact recovery condition of our algorithm. The BNSP for JOBS is stated as follows:

Definition 9 (BNSP for JOBS). A set of sensing matrices $\left\{\boldsymbol{A}_{1}, \boldsymbol{A}_{2}, \ldots, \boldsymbol{A}_{K}\right\}$ satisfies BNSP of order $s$ if $\quad \forall\left(\boldsymbol{v}_{1}, \boldsymbol{v}_{2}, \ldots, \boldsymbol{v}_{K}\right) \in \operatorname{Null}\left(\boldsymbol{A}_{1}\right) \times \operatorname{Null}\left(\boldsymbol{A}_{2}\right) \ldots \times$ $\operatorname{Null}\left(\boldsymbol{A}_{K}\right) \backslash\{(\mathbf{0}, \mathbf{0}, \ldots, \mathbf{0})\}$, such that for all $\mathcal{S}: \mathcal{S} \subset$ $\{1,2, \ldots, n\}, \operatorname{card}(\mathcal{S}) \leq s:\|\boldsymbol{V}[\mathcal{S}]\|_{1,2}<\left\|\boldsymbol{V}\left[\mathcal{S}^{c}\right]\right\|_{1,2}$.

Theorem 10 (Necessary and Sufficient Condition for JOBS). (i) $\mathbf{J}_{12}$ successfully recovers all the $s$-row sparse solution if and only if $\left\{\boldsymbol{A}\left[\mathcal{I}_{1}\right], \boldsymbol{A}\left[\mathcal{I}_{2}\right], \ldots, \boldsymbol{A}\left[\mathcal{I}_{K}\right]\right\}$ satisfies BNSP of the order of s. (ii) The solution is of the form $\boldsymbol{X}^{\star}=\left(\boldsymbol{x}^{\star}, \boldsymbol{x}^{\star}, \ldots, \boldsymbol{x}^{\star}\right)$, where $\boldsymbol{x}^{\star}$ is the unique solution to $\mathbf{P}_{1}$. Then, the JOBS solution $\boldsymbol{x}^{\boldsymbol{J}}$ is the average over columns of $\boldsymbol{X}^{\star}$, which is $\boldsymbol{x}^{\star}$.

Obtaining Definition 9 is straight forward. We prove it using the BNSP of the general $\ell_{p, 2}$ block norm stated in Appendix in Definition 19. Theorem 10 (i) can be obtained from BNSP in [17] and Theorem 10 (ii) can be derived by showing that $\boldsymbol{X}^{\star}$ is feasible and it achieves the lower bound of $\ell_{1,2}$ norm of feasible solutions. The BNSP of JOBS characterizes the existence and unique of solution and Theorem 10 establishes the correctness of JOBS.

\section{B. BRIP}

Since the BNSP is in general difficult to check, RIP, a more applicable quantity is derived. It useful in analyzing the error bounds for the noisy cases, where both sufficient conditions and error bounds are related to the RIP constant. We will show that the BRIP constant for JOBS can be decomposed to the maximum of RIP constants for all sensing matrices.

Let $\boldsymbol{A}^{\boldsymbol{J}}=$ block_diag $\left(\boldsymbol{A}\left[\mathcal{I}_{1}\right], \boldsymbol{A}[2], \ldots, \boldsymbol{A}\left[\mathcal{I}_{K}\right]\right)$ and $\mathcal{B}=$ $\left\{\mathcal{B}_{1}, \mathcal{B}_{2}, \ldots, \mathcal{B}_{n}\right\}$ be the group partition of $\{1,2, \ldots, n K\}$ that corresponds to row sparsity pattern. Let $\delta_{s \mid \mathcal{B}}$ denote row sparse BRIP constant of order $s$ and $\delta_{s}$ denote RIP constant of order $s$. The BRIP constant for JOBS is as follows.

Proposition 11 (BRIP for JOBS). For all $s \leq n, s \in \mathbb{Z}^{+}$

$$
\delta_{s \mid \mathcal{B}}\left(\boldsymbol{A}^{\boldsymbol{J}}\right)=\max _{j=1,2, \ldots, K} \delta_{s}\left(\boldsymbol{A}\left[\mathcal{I}_{j}\right]\right) .
$$

The proof of this proposition is elaborated in Appendix VIII-C. It is not so surprising that the BRIP of JOBS depends on the worst case among all $K$ choices of sub-matrices since smaller RIP constant indicates better recovering ability. More importantly, this result shows that the BRIP constant for JOBS can be decomposed into functions of standard RIP constant for each sub-matrix, which enables us to derive the sample complexity of our algorithm simply based on the sample complexity for $\ell_{1}$ minimization in Theorem 8

\section{Noisy Recovery Performance}

From previous analyses we can establish that if the BRIP constant of order $2 s$ is less than $\sqrt{2}-1$, it implies that $\left\{\boldsymbol{A}\left[\mathcal{I}_{1}\right], \boldsymbol{A}\left[\mathcal{I}_{2}\right], \ldots, \boldsymbol{A}\left[\mathcal{I}_{K}\right]\right\}$ satisfies BNSP of order $s$. Then, by Theorem 10 (ii), we know that the optimal solution to $\mathbf{J}_{\mathbf{1 2}}$ is the $s$-row sparse signal $\boldsymbol{X}^{\star}$ with every column being $\boldsymbol{x}^{\star}$. Similar to block sparse recovery bound in Theorem 7 , the reconstruction error is determined by the $s$-block sparse approximation error and the noise level. In the case when the true solution is exactly $s$-row sparse, it is relatively easy to analyze. For each realization, its performance can be analyzed through Theorem 
2 in [17]. Then to characterize typical performance of JOBS we use the tail bounds and obtain the following result.

Theorem 12 (JOBS: Error bound for $\left\|\boldsymbol{x}^{\star}\right\|_{0}=s$ ). Let $\boldsymbol{y}=$ $\boldsymbol{A} \boldsymbol{x}^{\star}+\boldsymbol{z},\|\boldsymbol{z}\|_{2}<\infty$. If $\delta_{2 s \mid \mathcal{B}}\left(\boldsymbol{A}^{\boldsymbol{J}}\right) \leq \delta<\sqrt{2}-1$ and the true solution is exactly $s$-sparse, then for any $\tau>0$, JOBS solution $x^{J}$ satisfies

$$
\begin{aligned}
& \mathbb{P}\left\{\left\|\boldsymbol{x}^{\boldsymbol{J}}-\boldsymbol{x}^{\star}\right\|_{2} \leq \mathcal{C}_{1}\left(\delta_{L}\right)\left(\sqrt{\frac{L}{m}}\|\boldsymbol{z}\|_{2}+\tau\right)\right\} \\
& \quad \geq 1-\exp \frac{-2 K \tau^{4}}{L\|\boldsymbol{z}\|_{\infty}^{4}} .
\end{aligned}
$$

In the more general case, when the sparsity level of $\boldsymbol{x}^{\star}$ possibly exceeds $s$, there is no guarantee that the non $s$-sparse part will be preserved by JOBS relaxation. Namely, let $\boldsymbol{X}^{\boldsymbol{J}}$ * denote the true solution for the noiseless row sparse recovery program: $\mathbf{J}_{\mathbf{1 2}}$, if BNSP of order greater than $s$ is not guaranteed to be satisfied, then it is not guarantee that $X^{J \star}=X^{\star}$. However, if $\boldsymbol{x}^{\star}$ is a near $s$-sparse, then $\boldsymbol{X}^{J \star}$ is not far away from $X^{\star}$. Since $X^{\sharp}$, recovered from $\mathbf{J}_{12}^{\epsilon^{J}}$, is close to $\boldsymbol{X}^{J \star}$ via the block sparse recovery bound, $\boldsymbol{X}^{\sharp}$ is close enough to $\boldsymbol{X}^{\star}$. This result is stated in the following theorem.

Theorem 13 (JOBS: Error bound for general signal recovery). Let $\boldsymbol{y}=\boldsymbol{A} \boldsymbol{x}^{\star}+\boldsymbol{z},\|\boldsymbol{z}\|_{2}<\infty$, If $\delta_{2 s \mid \mathcal{B}}\left(\boldsymbol{A}^{\boldsymbol{J}}\right) \leq \delta<\sqrt{2}-1$, then for any $\tau>0$, JOBS solution $x^{J}$ satisfies

$$
\begin{aligned}
& \mathbb{P}\left\{\left\|\boldsymbol{x}^{\boldsymbol{J}}-\boldsymbol{x}^{\star}\right\|_{2} \leq\|\boldsymbol{e}\|_{2}+\mathcal{C}_{1}\left(\delta_{L}\right)\left(\sqrt{\frac{L}{m}}\|\boldsymbol{A} \boldsymbol{e}+\boldsymbol{z}\|_{2}+\tau\right)\right\} \\
& \geq 1-\exp \frac{-2 K \tau^{4}}{L\left(\|\boldsymbol{A}\|_{\infty, 1}\|\boldsymbol{e}\|_{\infty}+\|\boldsymbol{z}\|_{\infty}\right)^{4}},
\end{aligned}
$$

where $\boldsymbol{e}$ is the s-sparse approximation error: $\boldsymbol{e}=\boldsymbol{x}^{\star}-\boldsymbol{x}_{0}$ with $\boldsymbol{x}_{0}$ being the top $s$ components of the true solution $\boldsymbol{x}^{\star}$, and $\left.\|\boldsymbol{A}\|_{\infty, 1}=\max _{i=1,2, \ldots, m}(\| \boldsymbol{a}[i]]^{\boldsymbol{T}} \|_{1}\right)$ denotes the largest $\ell_{1}$-norm of all rows of $\boldsymbol{A}$.

The error bound in Theorem 13 relates to $s$-sparse approximation error as well as the noise level, which is similar to $\ell_{1}$ minimization and block sparse recovery bounds. JOBS also introduces a relaxation error bounded by $\|\boldsymbol{e}\|_{2}$. The smaller the power of $e$, the smaller the upper bound. When $e=0, x^{\star}$ is exactly $s-$ sparse, then Theorem 13 reduces to Theorem 12 . From those two theorems, there are trade-offs for a good choice of bootstrap sample size $L$ and number of bootstrap samples $K$. The relationship of the bound and $L$ is the following: Because the BRIP constant decreases with the increasing $L$ and $\mathcal{C}_{1}(\delta)$ is a non-decreasing function of $\delta$, a larger $L$ results in a smaller $\mathcal{C}_{1}(\delta)$. The ratio $\sqrt{L / m}$, however, is smaller for smaller $L$. As for the number of estimates, the uncertainty in (13) decays exponentially with $K$, so a large $K$ is preferable in this sense.

\section{Noisy Recovery for Employing Bagging in Sparse Recovery}

We also derive the performance bound for employing Bagging scheme to sparse recovery problem, in which the final estimate is the average over multiple estimates solved individually from bootstrap samples. We give the theoretical results for the case that true signal $\boldsymbol{x}^{\star}$ is exactly $s$-sparse and the general case that it is not necessarily exactly $s$-sparse.

Theorem 14 (Bagging: Error bound for $\left\|\boldsymbol{x}^{\star}\right\|_{0}=s$ ). Let $\boldsymbol{y}=\boldsymbol{A} \boldsymbol{x}^{\star}+\boldsymbol{z},\|\boldsymbol{z}\|_{2}<\infty$, If under the assumption that, for $\left\{\mathcal{I}_{j}\right\}$ s that generates a set of sensing matrices $\boldsymbol{A}\left[\mathcal{I}_{1}\right], \boldsymbol{A}\left[\mathcal{I}_{2}\right], \ldots, \boldsymbol{A}\left[\mathcal{I}_{K}\right]$, there exists $\delta$ such that for all $j \in$ $\{1,2, \ldots, K\}, \delta_{2 s}\left(\boldsymbol{A}\left[\mathcal{I}_{j}\right]\right) \leq \delta<\sqrt{2}-1$. Let $\boldsymbol{x}^{\boldsymbol{B}}$ be the solution of Bagging, then for any $\tau>0, x^{B}$ satisfies

$$
\begin{aligned}
& \mathbb{P}\left\{\left\|\boldsymbol{x}^{\boldsymbol{B}}-\boldsymbol{x}^{\star}\right\|_{2} \leq \mathcal{C}_{1}\left(\delta_{L}\right)\left(\sqrt{\frac{L}{m}}\|\boldsymbol{z}\|_{2}+\tau\right)\right\} \\
& \quad \geq 1-\exp \frac{-2 K \tau^{4}}{L^{2}\|\boldsymbol{z}\|_{\infty}^{4}} .
\end{aligned}
$$

We also study the behavior of Bagging for general signal $\boldsymbol{x}^{\star},\left\|\boldsymbol{x}^{\star}\right\|_{0} \geq s$, in which the performance involves the $s$-sparse approximation error.

Theorem 15 (Bagging: Error bound for general signal recovery). Let $\boldsymbol{y}=\boldsymbol{A} \boldsymbol{x}^{\star}+\boldsymbol{z},\|\boldsymbol{z}\|_{2}<\infty$, If under the assumption that, for $\left\{\mathcal{I}_{j}\right\}$ s that generates a set of sensing matrices $\boldsymbol{A}\left[\mathcal{I}_{1}\right], \boldsymbol{A}\left[\mathcal{I}_{2}\right], \ldots, \boldsymbol{A}\left[\mathcal{I}_{K}\right]$, there exists $\delta$ such that for all $j \in\{1,2, \ldots, K\}, \delta_{2 s}\left(\boldsymbol{A}\left[\mathcal{I}_{j}\right]\right) \leq \delta<\sqrt{2}-1$. Let $\boldsymbol{x}^{B}$ be the solution of Bagging, then for any $\tau>0, x^{B}$ satisfies

$$
\begin{aligned}
& \mathbb{P}\left\{\left\|\boldsymbol{x}^{\boldsymbol{B}}-\boldsymbol{x}^{\star}\right\|_{2} \leq\left(\mathcal{C}_{0}\left(\delta_{L}\right) s^{-1 / 2}\|\boldsymbol{e}\|_{1}+\mathcal{C}_{1}\left(\delta_{L}\right)\left(\sqrt{\frac{L}{m}}\|\boldsymbol{z}\|_{2}+\tau\right)\right\}\right. \\
& \quad \geq 1-\exp \frac{-2 K \mathcal{C}_{1}^{4}(\delta) \tau^{4}}{\left(b^{\prime}\right)^{2}}
\end{aligned}
$$

where $b^{\prime}=\left(\mathcal{C}_{0}(\delta) s^{-1 / 2}\|\boldsymbol{e}\|_{1}+\mathcal{C}_{1}(\delta) \sqrt{L}\|\boldsymbol{z}\|_{\infty}\right)^{2}$.

Theorem 15 gives the performance bound for Bagging for general signal recovery without the $s$-sparse assumption, and it reduces to Theorem 14 when the $s$-sparse approximation error is zero $\|\boldsymbol{e}\|_{1}=0$. Both Theorem 14 and 15 above show that increasing the number of estimates $K$ improves the result, by increasing lower bound of the certainty of the same performance.

Here are some comments about the error bound for JOBS compared to Bagging. The RIP condition for Bagging is the same as the RIP condition for our algorithm, under the assumption that all submatrices $\boldsymbol{A}\left[\mathcal{I}_{j}\right]$ are well-behaved. When $\left\|\boldsymbol{x}^{\star}\right\|_{0}=s$, the bound in Bagging is worse than JOBS, since the certainty for algorithm is at least $1-\exp \frac{-2 K \tau^{4}}{L^{2}\|\boldsymbol{z}\|_{\infty}^{4}}$, compared to the error bound $1-\exp \frac{-2 K \tau^{4}}{L\|\boldsymbol{z}\|_{\infty}^{4}}$ in JOBS. With a squared $L$ instead of $L$, that term is smaller than the term in JOBS for the same choices of $L$ and $K$.

As for the general signal recovery bound of Bagging in Theorem 15, since the error bound for bagging does not contain the MMV relaxation error as the one in JOBS, however the tail bound involves more complicated terms. This bound is nontrivial comparing to the one to JOBS. Although the $s$-sparse assumption limits to exact $s$-sparse signal, for signals that are approximately $s$-sparse, or with low energy in the $s$-sparse approximation $\left(\|\boldsymbol{e}\|_{1}\right.$ is low), the behavior would be close to the exact $s$-sparse case. 
E. Sample Complexity for JOBS with i.i.d. Gaussian Sensing Matrices

Theorem 16 (Sample Complexity for JOBS). If the entries of the original sensing matrix $\boldsymbol{A}$ are i.i.d Gaussian or subGaussian, then for $d<L$, a small $\alpha$ and a small $\mu>0$, such that the minimum number of distinct elements across $\mathcal{I}_{i} s$ are bounded by $d: \mathbb{P}\left\{V\left(\mathcal{I}_{i}\right) \geq d\right\} \geq 1-\alpha(V(\mathcal{I})$ counts the number of distinct elements in multi-set $\mathcal{I})$, and if $d$ is in the order of $\mathcal{O}\left(2 s \ln (n / 2 s)+\ln K+\ln \left(\frac{(1-\alpha)^{K}}{(1-\alpha)^{K}-(1-\mu)}\right)\right)$, and the constant depending on $\alpha, \mu$ and $K, \mathbb{P}\left(\delta_{2 s \mid \mathcal{B}}\left(\boldsymbol{A}^{\boldsymbol{J}}\right)<\sqrt{2}-1\right) \geq$ $1-\mu$, and therefore, JOBS recovers the true $s$-sparse solution $\boldsymbol{X}^{\star}$ with at least a certain probability relates to $K, \alpha, \mu$.

In the sample complexity analysis, the $2 s \ln (n / 2 s)$ term coincides with the one from $\ell_{1}$ minimization. The terms associated with $K$ are non-decreasing with respect to $K$, which is introduced by the increasing uncertainty from taking a large number of bootstrap samples, resulting from the non-decreasing property of BRIP with adding extra sets of bootstrap samples shown in 11.

\section{PROOFS OF MAIN THEORETICAL RESULTS}

\section{A. Proof of Necessary and Sufficient condition: Theorem 10}

Theorem 10 (i) can be directly shown from the BNSP for block sparse minimization problems as in [17].

We show the procedure to prove Theorem 10 (ii). If BNSP of order $s$ is satisfied for $\left\{\boldsymbol{A}\left[\mathcal{I}_{1}\right], \boldsymbol{A}\left[\mathcal{I}_{2}\right], \ldots, \boldsymbol{A}\left[\mathcal{I}_{K}\right]\right\}$, then each submatrix $\boldsymbol{A}\left[\mathcal{I}_{j}\right]$ satisfies Null Space Property (NSP) of order $s$. The detailed proof is in Appendix VIII-B in proving Lemma 20. Consequently, for all $j=1,2, \ldots, K$, let $\boldsymbol{x}^{\star}$ be the optimal solution:

$$
\boldsymbol{x}^{\star}=\underset{\boldsymbol{x}_{j}}{\arg \min }\left\|\boldsymbol{x}_{j}\right\|_{1} \text { s.t. } \boldsymbol{y}\left[\mathcal{I}_{j}\right]=\boldsymbol{A}\left[\mathcal{I}_{j}\right] \boldsymbol{x}_{j} .
$$

For $\boldsymbol{X}$ a feasible solution, consider its $\ell_{1,2}$ norm, we have:

$$
\|\boldsymbol{X}\|_{1,2}=\sum_{i=1}^{n}\left(\sum_{j=1}^{K}\left(x_{i j}^{2}\right)\right)^{1 / 2}=\sqrt{K} \sum_{i=1}^{n}\left(\frac{1}{K} \sum_{j=1}^{K}\left(x_{i j}^{2}\right)\right)^{1 / 2}
$$

By concavity of square root, we have

$$
\begin{aligned}
& \geq \sqrt{K} \sum_{i=1}^{n} \frac{1}{K} \sum_{j=1}^{K} \sqrt{x_{i j}^{2}}=\sqrt{K} \frac{1}{K} \sum_{j=1}^{K} \sum_{i=1}^{n}\left|x_{i j}\right| \\
& \geq \sqrt{K} \frac{1}{K} \sum_{j=1}^{K} \min _{\substack{\boldsymbol{x}_{j}: x_{1 j}, \ldots, x_{n j} \\
\boldsymbol{A}\left[\mathcal{I}_{j}\right] \boldsymbol{x}_{j}=\boldsymbol{y}\left[\mathcal{I}_{j}\right]}} \sum_{i=1}^{n}\left|x_{i j}\right| \\
& =\sqrt{K} \frac{1}{K} \sum_{j=1}^{K} \min _{\boldsymbol{x}_{j}: \boldsymbol{A}\left[\mathcal{I}_{j}\right] \boldsymbol{x}_{j}=\boldsymbol{y}\left[\mathcal{I}_{j}\right]}\left\|\boldsymbol{x}_{j}\right\|_{1} \\
& =\sqrt{K}\left\|\boldsymbol{x}^{\star}\right\|_{1}
\end{aligned}
$$

$\boldsymbol{X}^{\star}=\left(\boldsymbol{x}^{\star}, \boldsymbol{x}^{\star}, \ldots, \boldsymbol{x}^{\star}\right)$ is a feasible solution and $\left\|\boldsymbol{X}^{\star}\right\|_{1,2}=$ $\left\|\left(\boldsymbol{x}^{\star}, \boldsymbol{x}^{\star}, \ldots, \boldsymbol{x}^{\star}\right)\right\|_{1,2}=\sqrt{K}\left\|\boldsymbol{x}^{\star}\right\|_{1}$, and it achieves the lower bound. By uniqueness from (i), we can concluded that $\boldsymbol{X}^{\star}$ is the unique solution. The JOBS solution takes average over columns of multiple estimates. Since the average of $\boldsymbol{X}^{\star}$ is $\boldsymbol{x}^{\star}$, we prove that JOBS returns the correct answer.
B. Proof of Theorem 12, performance bound of for exactly s-sparse

If the true solution is exactly $s$-sparse, the sparse approximation error is zero. Then the noise level of performance only relates to measurements noise. For $\ell_{1}$ minimization, $z$ is the noise vector and we use matrix $\boldsymbol{Z}=\left(\boldsymbol{z}\left[\mathcal{I}_{1}\right], \boldsymbol{z}\left[\mathcal{I}_{2}\right], \ldots, \boldsymbol{z}\left[\mathcal{I}_{K}\right]\right)$ to denote the noise matrix in JOBS. We bound the distance of $\|\boldsymbol{Z}\|_{2,2}$ to its expected value using Hoeffidings' inequalities stated in [33] by Hoeffding in 1963.

Theorem 17 (Hoeffdings' Inequalities). Let $X_{1}, \ldots, X_{n}$ be independent bounded random variables such that $X_{i}$ falls in the interval $\left[a_{i}, b_{i}\right]$ with probability one. Denote their sum by $S_{n}=\sum_{i=1}^{n} X_{i}$. Then for any $\epsilon>0$, we have:

$$
\begin{aligned}
& \mathbb{P}\left\{S_{n}-\mathbb{E} \mathcal{S}_{n} \geq \epsilon\right\} \leq \exp \frac{-2 \epsilon^{2}}{\sum_{i=1}^{n}\left(b_{i}-a_{i}\right)^{2}} \text { and } \\
& \mathbb{P}\left\{S_{n}-\mathbb{E} \mathcal{S}_{n} \leq-\epsilon\right\} \leq \exp \frac{-2 \epsilon^{2}}{\sum_{i=1}^{n}\left(b_{i}-a_{i}\right)^{2}}
\end{aligned}
$$

Here, the whole noise vector is $\boldsymbol{z}=\boldsymbol{A x}-\boldsymbol{y}=$ $(z[1], z[2], \ldots, z[m])^{T},\|\boldsymbol{z}\|_{\infty}=\max _{i \in\{1,2, \ldots, m\}}|z[i]|<\infty$. We consider the matrix $\boldsymbol{Z} \circ \boldsymbol{Z}=\left(\xi_{j i}\right)$, where $\circ$ is the entry-wise product. The quantity that we are interested in $\|\boldsymbol{Z}\|_{2,2}$ is the sum of all entries in $\boldsymbol{Z} \circ \boldsymbol{Z}$. Each element in this matrix $\boldsymbol{Z} \circ \boldsymbol{Z}$ is drawn i.i.d from the squares of entries in $z$ : $\{z[1], z[2], \ldots, z[m]\}$ with equal probability. Let $\Xi$ be the underlining random variable and $\Xi$ obeys a discrete uniform distribution

$$
\mathbb{P}\left(\Xi=z^{2}[i]\right)=\frac{1}{m}, i=1,2, \ldots, m
$$

The lower and upper bound of $\Xi$ is

$$
0 \leq \min _{i} z^{2}[i] \leq \Xi \leq\|\boldsymbol{z}\|_{\infty}^{2}
$$

We use zero as lower bound for $\Xi$ instead of the minimun value to simplify the terms. The expected power of $Z$ is

$$
\mathbb{E}\|\boldsymbol{Z}\|_{2,2}^{2}=\frac{K L}{m}\|\boldsymbol{z}\|_{2}^{2}
$$

Then applying Hoeffdings' inequality (17), for any $\tau>0$, we have:

$$
\mathbb{P}\left\{\|\boldsymbol{Z}\|_{2,2}^{2}-\mathbb{E}\|\boldsymbol{Z}\|_{2,2}^{2}-\tau \leq 0\right\} \geq 1-\exp \frac{-2 \tau^{2}}{K L\|\boldsymbol{z}\|_{\infty}^{4}} .
$$

Let $\boldsymbol{X}^{\sharp}$ be the solution of $\mathbf{J}_{\mathbf{1 2}}^{\epsilon^{J}}$, and by Theorem 7 :

$$
\mathbb{P}\left\{\left\|\boldsymbol{X}^{\sharp}-\boldsymbol{X}^{\star}\right\|_{2,2}^{2}-\mathcal{C}_{1}^{2}(\delta)\|\boldsymbol{Z}\|_{2,2}^{2} \leq 0\right\}=1 \text {. }
$$

Let $\Delta$ be the difference between the solution to the truth solution scaled by $\mathcal{C}_{1}$ constant: $\Delta=\frac{1}{\mathcal{C}_{1}(\delta)}\left\|\boldsymbol{X}^{\sharp}-\boldsymbol{X}^{\star}\right\|_{2,2}$ and (23) becomes

$$
\mathbb{P}\left\{\Delta-\|\boldsymbol{Z}\|_{2,2} \leq 0\right\}=1
$$


Since $Z$ depends on the choice of $\mathcal{I}_{1}, \mathcal{I}_{2}, \ldots, \mathcal{I}_{K}$, we derive the typical performance by studying the distance of the solution to the expected noise level of JOBS.

$$
\begin{aligned}
& \mathbb{P}\left\{\Delta^{2}-\mathbb{E}\|\boldsymbol{Z}\|_{2,2}^{2}-\tau^{2} \leq 0\right\} \\
& =\mathbb{P}\left\{\Delta^{2}-\|\boldsymbol{Z}\|_{2,2}^{2}+\|\boldsymbol{Z}\|_{2,2}^{2}-\mathbb{E}\|\boldsymbol{Z}\|_{2,2}^{2}-\tau^{2} \leq 0\right\} \\
& \geq \mathbb{P}\left\{\Delta^{2}-\|\boldsymbol{Z}\|_{2,2}^{2} \leq 0,\|\boldsymbol{Z}\|_{2,2}^{2}-\mathbb{E}\|\boldsymbol{Z}\|_{2,2}^{2}-\tau^{2} \leq 0\right\}
\end{aligned}
$$

(The first and the second parts are independent)

$$
=\mathbb{P}\left\{\Delta^{2}-\|\boldsymbol{Z}\|_{2,2}^{2} \leq 0\right\} \mathbb{P}\left\{\|\boldsymbol{Z}\|_{2,2}^{2}-\mathbb{E}\|\boldsymbol{Z}\|_{2,2}^{2}-\tau^{2} \leq 0\right\}
$$

(using 24) and (22))

$$
\geq 1-\exp \frac{-2 \tau^{4}}{K L\|\boldsymbol{z}\|_{\infty}^{4}} \text {. }
$$

This procedure gives:

$$
\mathbb{P}\left\{\Delta^{2} \leq \mathbb{E}\|\boldsymbol{Z}\|_{2,2}^{2}+\tau^{2}\right\} \geq 1-\exp \frac{-2 \tau^{4}}{K L\|\boldsymbol{z}\|_{\infty}^{4}} .
$$

We bound the squared error as the following:

$$
\begin{aligned}
& \mathbb{P}\left\{\Delta \leq\left(\mathbb{E}\|\boldsymbol{Z}\|_{2,2}^{2}\right)^{1 / 2}+\tau\right\} \\
= & \mathbb{P}\left\{\Delta^{2} \leq \mathbb{E}\|\boldsymbol{Z}\|_{2,2}^{2}+\tau^{2}+2 \tau\left(\mathbb{E}\|\boldsymbol{Z}\|_{2,2}^{2}\right)^{1 / 2}\right\} \\
\geq & \mathbb{P}\left\{\Delta^{2} \leq \mathbb{E}\|\boldsymbol{Z}\|_{2,2}^{2}+\tau^{2}\right\} .
\end{aligned}
$$

Combining 25] and 26, we have:

$$
\mathbb{P}\left\{\Delta \leq\left(\mathbb{E}\|\boldsymbol{Z}\|_{2,2}^{2}\right)^{1 / 2}+\tau\right\} \geq 1-\exp \frac{-2 \tau^{4}}{K L\|\boldsymbol{z}\|_{\infty}^{4}} .
$$

Since $f(\boldsymbol{x})=\left\|\boldsymbol{x}-\boldsymbol{x}^{\star}\right\|_{2}^{2}$ is convex, we can apply Jensens' inequality:

$$
\left\|\frac{1}{K} \sum_{j=1}^{K} \boldsymbol{x}_{j}^{\sharp}-\boldsymbol{x}^{\star}\right\|_{2}^{2} \leq \frac{1}{K} \sum_{j=1}^{K}\left\|\boldsymbol{x}_{j}^{\sharp}-\boldsymbol{x}^{\star}\right\|_{2}^{2} .
$$

The JOBS estimate is averaged over columns of all estimates: $\boldsymbol{x}^{\boldsymbol{J}}=\frac{1}{K} \sum_{j=1}^{K} \boldsymbol{x}_{j}^{\sharp}$. Therefore, equation 28 is essentially

$$
\mathbb{P}\left\{\left\|\boldsymbol{x}^{\boldsymbol{J}}-\boldsymbol{x}^{\star}\right\|_{2}^{2}-\frac{1}{K}\left\|\boldsymbol{X}^{\sharp}-\boldsymbol{X}^{\star}\right\|_{2,2}^{2} \leq 0\right\}=1 .
$$

Now we consider the typical performance of the JOBS solution:

$$
\begin{aligned}
& \mathbb{P}\left\{\left\|\boldsymbol{x}^{\boldsymbol{J}}-\boldsymbol{x}^{\star}\right\|_{2}-\frac{\mathcal{C}_{1}(\delta)}{\sqrt{K}}\left(\left(\mathbb{E}\|\boldsymbol{Z}\|_{2,2}^{2}\right)^{1 / 2}+\tau\right) \leq 0\right\} \\
= & \mathbb{P}\left\{\left\|\boldsymbol{x}^{\boldsymbol{J}}-\boldsymbol{x}^{\star}\right\|_{2}-\frac{1}{\sqrt{K}}\left\|\boldsymbol{X}^{\sharp}-\boldsymbol{X}^{\star}\right\|_{2}\right. \\
& \left.+\frac{1}{\sqrt{K}}\left\|\boldsymbol{X}^{\sharp}-\boldsymbol{X}^{\star}\right\|_{2}-\frac{\mathcal{C}_{1}(\delta)}{\sqrt{K}}\left(\left(\mathbb{E}\|\boldsymbol{Z}\|_{2,2}^{2}\right)^{1 / 2}+\tau\right) \leq 0\right\} \\
\geq & \mathbb{P}\left\{\left\|\boldsymbol{x}^{\boldsymbol{J}}-\boldsymbol{x}^{\star}\right\|_{2}-\frac{1}{\sqrt{K}}\left\|\boldsymbol{X}^{\sharp}-\boldsymbol{X}^{\star}\right\|_{2} \leq 0,\right. \\
& \left.\Delta \leq\left(\mathbb{E}\|\boldsymbol{Z}\|_{2,2}^{2}\right)^{1 / 2}+\tau\right\} \\
= & \mathbb{P}\left\{\left\|\boldsymbol{x}^{\boldsymbol{J}}-\boldsymbol{x}^{\star}\right\|_{2}-\frac{1}{\sqrt{K}}\left\|\boldsymbol{X}^{\sharp}-\boldsymbol{X}^{\star}\right\|_{2} \leq 0\right\} \\
& \mathbb{P}\left\{\Delta \leq\left(\mathbb{E}\|\boldsymbol{Z}\|_{2,2}^{2}\right)^{1 / 2}+\tau\right\} \quad \quad \quad \text { by }[29 \text { and 27]) } \\
\geq & 1-\exp \frac{-2 \tau^{4}}{K L\|\boldsymbol{z}\|_{\infty}^{4}} .
\end{aligned}
$$

Then Plug in the expected noise level derived in (21),

$$
\begin{aligned}
& \mathbb{P}\left\{\left\|\boldsymbol{x}^{J}-\boldsymbol{x}^{\star}\right\|_{2} \leq \mathcal{C}_{1}(\delta)\right.\left.\left(\sqrt{\frac{L}{m}}\|\boldsymbol{z}\|_{2}+\frac{\tau}{\sqrt{K}}\right)\right\} \\
& \geq 1-\exp \frac{-2 \tau^{4}}{K L\|\boldsymbol{z}\|_{\infty}^{4}} .
\end{aligned}
$$

and replacing $\tau / \sqrt{K}$ with $\tau$, the quantity on the right hand side of the equation then becomes $1-\exp \frac{-2 K \tau^{4}}{L\|z\|_{\infty}^{4}}$ and we prove the theorem.

\section{Proof of Theorem 13}

Now we consider the case that the BNSP is only satisfied for order $s$ whereas there is no $s$-sparse assumption on the true solution. Therefore, the algorithm can only guarantee the correctness of the $s$-row sparse part and our best hope is to recover the best $s$-row sparse approximation of the true solution. Let $\boldsymbol{x}_{0}$ be the best $s$-row sparse approximation of the true solution $\boldsymbol{x}^{\star}$ and $\boldsymbol{e}$ denote the difference $\boldsymbol{e}=\boldsymbol{x}^{\star}-\boldsymbol{x}_{0}$. We rewrite the measurements to include the $s$-row sparse approximation error as part of noise: for $j=1,2, \ldots, K$,

$$
\begin{aligned}
\boldsymbol{y}\left[\mathcal{I}_{j}\right] & =\boldsymbol{A}\left[\mathcal{I}_{j}\right] \boldsymbol{x}^{\star}+\boldsymbol{z}\left[\mathcal{I}_{j}\right]=\boldsymbol{A}\left[\mathcal{I}_{j}\right]\left(\boldsymbol{x}_{0}+\left(\boldsymbol{x}^{\star}-\boldsymbol{x}_{0}\right)\right)+\boldsymbol{z}\left[\mathcal{I}_{j}\right] \\
& =\boldsymbol{A}\left[\mathcal{I}_{j}\right] \boldsymbol{x}_{0}+\widetilde{\boldsymbol{z}}_{j},
\end{aligned}
$$

where $\widetilde{\boldsymbol{z}}_{j}=\boldsymbol{A}\left[\mathcal{I}_{j}\right]\left(\boldsymbol{x}^{\star}-\boldsymbol{x}_{0}\right)+\boldsymbol{z}\left[\mathcal{I}_{j}\right]=\boldsymbol{A}\left[\mathcal{I}_{j}\right] \boldsymbol{e}+\boldsymbol{z}\left[\mathcal{I}_{j}\right]$.

To bound the distance of solution of $\mathbf{J}_{12}^{\epsilon^{J}}$ : $X^{\sharp}$ to the true solution $\boldsymbol{X}^{\star}$, we use the exactly $s$ row sparse matrix $\boldsymbol{X}_{\mathbf{0}}=\left(\boldsymbol{x}_{\mathbf{0}}, \boldsymbol{x}_{\mathbf{0}}, \ldots, \boldsymbol{x}_{\mathbf{0}}\right)$ as the bridge. Since $\boldsymbol{e}=\boldsymbol{x}^{\star}-\boldsymbol{x}_{\mathbf{0}}$, we have: $\boldsymbol{X}^{\star}-\boldsymbol{X}_{\mathbf{0}}=(\boldsymbol{e}, \boldsymbol{e}, \ldots, \boldsymbol{e})$ and hence $\left\|\boldsymbol{X}_{\mathbf{0}}-\boldsymbol{X}^{\star}\right\|_{2,2}=$ $\sqrt{K}\|\boldsymbol{e}\|_{2}$. Then the distance of $\boldsymbol{X}^{\sharp}$ to the true solution $\boldsymbol{X}^{\star}$ can be decomposed into two parts:

$$
\begin{aligned}
& \left\|\boldsymbol{X}^{\sharp}-\boldsymbol{X}^{\star}\right\|_{2,2}=\left\|\boldsymbol{X}^{\sharp}-\boldsymbol{X}_{\mathbf{0}}+\boldsymbol{X}_{\mathbf{0}}-\boldsymbol{X}^{\star}\right\|_{2,2} \\
& \leq\left\|\boldsymbol{X}^{\sharp}-\boldsymbol{X}_{\mathbf{0}}\right\|_{2,2}+\left\|\boldsymbol{X}_{\mathbf{0}}-\boldsymbol{X}^{\star}\right\|_{2,2} \\
& =\left\|\boldsymbol{X}^{\sharp}-\boldsymbol{X}_{\mathbf{0}}\right\|_{2,2}+\sqrt{K}\|\boldsymbol{e}\|_{2} .
\end{aligned}
$$

To bound the first term in 32 : $\left\|\boldsymbol{X}^{\sharp}-\boldsymbol{X}_{\mathbf{0}}\right\|_{2,2}$, we will use the recovery guarantee from the row sparse recovery result in Theorem 7 , which gives a upper bound of this term associated with the power of the noise matrix $\widetilde{\boldsymbol{Z}}=\left(\widetilde{\boldsymbol{z}}_{1}, \widetilde{\boldsymbol{z}}_{2}, \ldots, \widetilde{\boldsymbol{z}}_{K}\right)$ :

$$
\begin{aligned}
\|\widetilde{\boldsymbol{Z}}\|_{2,2}^{2} & =\sum_{j=1}^{K}\left\|\widetilde{\boldsymbol{z}}_{j}\right\|_{2}^{2}=\sum_{j=1}^{K}\left\|\boldsymbol{A}\left[\mathcal{I}_{j}\right] \boldsymbol{e}+\boldsymbol{z}\left[\mathcal{I}_{j}\right]\right\|_{2}^{2} \\
& =\sum_{j=1}^{K} \sum_{i \in \mathcal{I}_{j}}(\langle\boldsymbol{a}[i], \boldsymbol{e}\rangle+z[i])^{2} .
\end{aligned}
$$

Then if we let $\widetilde{\Xi}=(\langle\boldsymbol{a}[i], \boldsymbol{e}\rangle+z[i])^{2}$ with $\boldsymbol{a}[i], \boldsymbol{z}[i]$ generated uniformly from all rows of $\boldsymbol{A}$ and $\boldsymbol{z}$. Since $\widetilde{\Xi}$ is non-negative, $\Xi \geq 0$, the lower bound is 0 . The upper bound is derived using Hölders inequality:

$$
\begin{aligned}
& \widetilde{\Xi}=(\langle\boldsymbol{a}[i], \boldsymbol{e}\rangle+z[i])^{2} \leq\left(\|\boldsymbol{a}[i] \cdot \boldsymbol{e}\|_{1}+\|\boldsymbol{z}\|_{\infty}\right)^{2} \\
& \leq\left(\left\|\boldsymbol{a}[i]^{T}\right\|_{1}\|\boldsymbol{e}\|_{\infty}+\|\boldsymbol{z}\|_{\infty}\right)^{2} \\
& \leq\left(\max _{i}\left\|\boldsymbol{a}[i]^{T}\right\|_{1}\|\boldsymbol{e}\|_{\infty}+\|\boldsymbol{z}\|_{\infty}\right)^{2} \\
& =\left(\|\boldsymbol{A}\|_{\infty}, 1\|\boldsymbol{e}\|_{\infty}+\|\boldsymbol{z}\|_{\infty}\right)^{2},
\end{aligned}
$$


where $\|\boldsymbol{A}\|_{\infty, 1}=\max _{i \in[m]}\left\|\boldsymbol{a}[i]^{T}\right\|_{1}$. Since $\boldsymbol{A}$ is deterministic with all bounded entries, $\|\boldsymbol{A}\|_{\infty, 1}$ is bounded.

From 33 , the expectation of $\|\widetilde{\boldsymbol{Z}}\|_{2,2}^{2}$ is

$$
\begin{aligned}
& \mathbb{E}\|\widetilde{\boldsymbol{Z}}\|_{2,2}^{2}=\sum_{j=1}^{K} \sum_{i \in \mathcal{I}_{j}} \mathbb{E}(\langle\boldsymbol{a}[i], \boldsymbol{e}\rangle)^{2}+2 \mathbb{E} z[i]\langle\boldsymbol{a}[i], \boldsymbol{e}\rangle \\
& +\mathbb{E} z[i]^{2}=\frac{K L}{m}\|\boldsymbol{A} \boldsymbol{e}+\boldsymbol{z}\|_{2}^{2} .
\end{aligned}
$$

Obtaining the the lower and upper bound of $\widetilde{\Xi}$, we can apply Hoeffdings' inequality to get the tail bound of $\|\widetilde{\boldsymbol{Z}}\|_{2,2}^{2}$, which can be written as, for any $\tau>0$,

$$
\begin{aligned}
& \mathbb{P}\left\{\|\widetilde{\boldsymbol{Z}}\|_{2,2}^{2}-\mathbb{E}\|\widetilde{\boldsymbol{Z}}\|_{2,2}^{2}-\tau \leq 0\right\} \\
& \geq 1-\exp \frac{-2 \tau^{2}}{K L\left(\|\boldsymbol{A}\|_{\infty, 1}\|\boldsymbol{e}\|_{\infty}+\|\boldsymbol{z}\|_{\infty}\right)^{4}}
\end{aligned}
$$

Then similar to prove Theorem 12 , here we consider the distance from the recovered solution $\boldsymbol{X}^{\sharp}$ to the exactly $s$-row sparse solution $\boldsymbol{X}_{\mathbf{0}}$. Let $\widetilde{\Delta}$ be $\widetilde{\Delta}=\frac{1}{\mathcal{C}_{1}(\delta)}\left\|\boldsymbol{X}^{\sharp}-\boldsymbol{X}_{\mathbf{0}}\right\|_{2,2}$ and according to Theorem 7, we have

$$
\mathbb{P}\left\{\|\widetilde{\Delta}-\| \widetilde{\boldsymbol{Z}} \|_{2,2} \leq 0\right\}=1 .
$$

Combing (36) and (37), we are able to conclude

$$
\begin{aligned}
& \mathbb{P}\left\{\widetilde{\Delta}^{2}-\mathbb{E}\|\widetilde{\boldsymbol{Z}}\|_{2,2}^{2}-\tau^{2} \leq 0\right\} \\
& =\mathbb{P}\left\{\widetilde{\Delta}^{2}-\|\boldsymbol{Z}\|_{2,2}^{2}+\|\widetilde{\boldsymbol{Z}}\|_{2,2}^{2}-\mathbb{E}\|\widetilde{\boldsymbol{Z}}\|_{2,2}^{2}-\tau^{2} \leq 0\right\} \\
& \geq \mathbb{P}\left\{\widetilde{\Delta}^{2}-\|\boldsymbol{Z}\|_{2,2}^{2} \leq 0,\|\widetilde{\boldsymbol{Z}}\|_{2,2}^{2}-\mathbb{E}\|\widetilde{\boldsymbol{Z}}\|_{2,2}^{2}-\tau^{2} \leq 0\right\} \\
& =\mathbb{P}\left\{\widetilde{\Delta}^{2}-\|\boldsymbol{Z}\|_{2,2}^{2} \leq 0\right\} \mathbb{P}\left\{\|\widetilde{\boldsymbol{Z}}\|_{2,2}^{2}-\mathbb{E}\|\widetilde{\boldsymbol{Z}}\|_{2,2}^{2}-\tau^{2} \leq 0\right\} \\
& \geq 1-\exp \frac{-2 \tau^{4}}{K L\left(\|\boldsymbol{A}\|_{\infty, 1}\|\boldsymbol{e}\|_{\infty}+\|\boldsymbol{z}\|_{\infty}\right)^{4}} .
\end{aligned}
$$

We bound the expected square root of noise power:

$$
\begin{aligned}
\mathbb{P} & \left.\left\{\widetilde{\Delta} \leq\left(\mathbb{E}\|\widetilde{\boldsymbol{Z}}\|_{2,2}^{2}\right)^{1 / 2}+\tau\right\} \quad \text { by } 26\right) \\
& \geq \mathbb{P}\left\{\widetilde{\Delta}^{2} \leq \mathbb{E}\|\widetilde{\boldsymbol{Z}}\|_{2,2}^{2}+\tau^{2}\right\} \\
& \geq 1-\exp \frac{-2 \tau^{4}}{K L\left(\|\boldsymbol{A}\|_{\infty, 1}\|\boldsymbol{e}\|_{\infty}+\|\boldsymbol{z}\|_{\infty}\right)^{4}} .
\end{aligned}
$$

Then, the final JOBS estimates $\boldsymbol{x}^{\boldsymbol{J}}$ is $\boldsymbol{x}^{\boldsymbol{J}}=\frac{1}{K} \sum_{j=1}^{K} \boldsymbol{x}_{j}^{\sharp}$ and by (29), we have:

$$
\begin{aligned}
\left\|\boldsymbol{x}^{\boldsymbol{J}}-\boldsymbol{x}^{\star}\right\|_{2} & \leq \frac{1}{\sqrt{K}}\left\|\boldsymbol{X}^{\sharp}-\boldsymbol{X}^{\star}\right\|_{2,2} \\
(\text { by } 32 \text { ) }) & \leq \frac{1}{\sqrt{K}}\left\|\boldsymbol{X}^{\sharp}-\boldsymbol{X}_{\mathbf{0}}\right\|_{2,2}+\|\boldsymbol{e}\|_{2}=\frac{\mathcal{C}_{1}(\delta) \widetilde{\Delta}}{\sqrt{K}}+\|\boldsymbol{e}\|_{2}
\end{aligned}
$$

Combing the results from (38), (39), we have:

$$
\begin{aligned}
& \mathbb{P}\left\{\left\|\boldsymbol{x}^{\boldsymbol{J}}-\boldsymbol{x}^{\star}\right\|_{2} \leq \frac{\mathcal{C}_{1}(\delta)\left(\left(\mathbb{E}\|\widetilde{\boldsymbol{Z}}\|_{2,2}^{2}\right)^{1 / 2}+\tau\right)}{\sqrt{K}}+\|\boldsymbol{e}\|_{2}\right\} \\
& \geq \mathbb{P}\left\{\frac{\mathcal{C}_{1}(\delta) \widetilde{\Delta}}{\sqrt{K}}+\|\boldsymbol{e}\|_{2} \leq \frac{\mathcal{C}_{1}(\delta)\left(\left(\mathbb{E}\|\widetilde{\boldsymbol{Z}}\|_{2,2}^{2}\right)^{1 / 2}+\tau\right)}{\sqrt{K}}+\|\boldsymbol{e}\|_{2}\right\} \\
& =\mathbb{P}\left\{\widetilde{\Delta} \leq\left(\mathbb{E}\|\widetilde{\boldsymbol{Z}}\|_{2,2}^{2}\right)^{1 / 2}+\tau\right\} \\
& \geq 1-\exp \frac{-2 k \tau^{4}}{\left(\|\boldsymbol{A}\|_{\infty, 1}\|\boldsymbol{e}\|_{\infty}+\|\boldsymbol{z}\|_{\infty}\right)^{4}} .
\end{aligned}
$$

Then plug in the expected noise level derived in 35,

$$
\begin{aligned}
\mathbb{P} & \left\{\left\|\boldsymbol{x}^{\boldsymbol{J}}-\boldsymbol{x}^{\star}\right\|_{2}\right. \\
& \left.\leq \mathcal{C}_{1}(\delta)\left(\sqrt{\frac{L}{m}}\|\boldsymbol{A} \boldsymbol{e}+\boldsymbol{z}\|_{2}+\frac{\tau}{\sqrt{K}}\right)+\|\boldsymbol{e}\|_{2}\right\} \\
& \geq 1-\exp \frac{-2 \tau^{4}}{K L\left(\|\boldsymbol{A}\|_{\infty, 1}\|\boldsymbol{e}\|_{\infty}+\|\boldsymbol{z}\|_{\infty}\right)^{4}} .
\end{aligned}
$$

and replacing $\tau$ with $\tau / \sqrt{K}$, the quantity on the right hand side of the equation then becomes $1-\exp \frac{-2 K \tau^{4}}{L\left(\|\boldsymbol{A}\|_{\infty, 1}\|\boldsymbol{e}\|_{\infty}+\|\boldsymbol{z}\|_{\infty}\right)^{4}}$ and we prove the theorem.

\section{Proof of Theorem 14 performance bound of bagging for exactly s-sparse signal recovery}

Let $x^{B}$ be the solution of the bagging scheme, and it is an average over individual solved problems $\boldsymbol{x}_{1}^{\boldsymbol{B}}, \boldsymbol{x}_{2}^{\boldsymbol{B}}, \ldots, \boldsymbol{x}_{K}^{\boldsymbol{B}}$ : $\boldsymbol{x}^{B}=\frac{1}{K} \sum_{j=1}^{K} \boldsymbol{x}_{j}^{B}$. we consider the distance to the true solution $\boldsymbol{x}^{\star}$ to each estimate separately. Here, the desired upper bound is the square root of the expected power of each noise vector: $\left(\mathbb{E} \boldsymbol{z}[\mathcal{I}] \|_{2}^{2}\right)^{1 / 2}=\sqrt{\frac{L}{m}}\|\boldsymbol{z}\|_{2}$, where $\mathcal{I}$ is a multi-set of size $L$ with each element randomly sampled from $\{1,2, \ldots, m\}$. For $\tau>0$, we consider:

$$
\begin{aligned}
& \mathbb{P}\left\{\left\|\boldsymbol{x}^{\boldsymbol{B}}-\boldsymbol{x}^{\star}\right\|_{2}-\mathcal{C}_{1}(\delta)\left(\left(\mathbb{E}\|\boldsymbol{z}[\mathcal{I}]\|_{2}^{2}\right)^{1 / 2}+\tau\right) \leq 0\right\} \\
& =\mathbb{P}\left\{\left\|\boldsymbol{x}^{B}-\boldsymbol{x}^{\star}\right\|_{2}-\mathcal{C}_{1}(\delta)\left(\left(\left(\mathbb{E}\|\boldsymbol{z}[\mathcal{I}]\|_{2}^{2}\right)^{1 / 2}+\tau\right)^{2}\right)^{1 / 2} \leq 0\right\} \\
& \geq \mathbb{P}\left\{\left\|\boldsymbol{x}^{B}-\boldsymbol{x}^{\star}\right\|_{2}-\mathcal{C}_{1}(\delta)\left(\mathbb{E}\|\boldsymbol{z}[\mathcal{I}]\|_{2}^{2}+\tau^{2}\right)^{1 / 2} \leq 0\right\} \\
& =\mathbb{P}\left\{\left\|\boldsymbol{x}^{\boldsymbol{B}}-\boldsymbol{x}^{\star}\right\|_{2}^{2}-\mathcal{C}_{1}^{2}(\delta)\left(\mathbb{E}\|\boldsymbol{z}[\mathcal{I}]\|_{2}^{2}+\tau^{2}\right) \leq 0\right\}
\end{aligned}
$$

Consider using the average of errors for each estimate: $\frac{1}{K} \sum_{j=1}^{K}\left\|\boldsymbol{x}_{j}^{B}-\boldsymbol{x}^{\star}\right\|_{2}^{2}$, we have

$$
\begin{aligned}
& =\mathbb{P}\left\{\left\|\boldsymbol{x}^{\boldsymbol{B}}-\boldsymbol{x}^{\star}\right\|_{2}^{2}-\frac{1}{K} \sum_{j=1}^{K}\left\|\boldsymbol{x}_{j}^{\boldsymbol{B}}-\boldsymbol{x}^{\star}\right\|_{2}^{2}\right. \\
& \left.+\frac{1}{K} \sum_{j=1}^{K}\left\|\boldsymbol{x}_{j}^{\boldsymbol{B}}-\boldsymbol{x}^{\star}\right\|_{2}^{2}-\mathcal{C}_{1}^{2}(\delta)\left(\mathbb{E}\|\boldsymbol{z}[\mathcal{I}]\|_{2}^{2}+\tau^{2}\right) \leq 0\right\} \\
& \geq \mathbb{P}\left\{\left\|\boldsymbol{x}^{\boldsymbol{B}}-\boldsymbol{x}^{\star}\right\|_{2}^{2}-\frac{1}{K} \sum_{j=1}^{K}\left\|\boldsymbol{x}_{j}^{\boldsymbol{B}}-\boldsymbol{x}^{\star}\right\|_{2}^{2} \leq 0,\right. \\
& \left.\frac{1}{K} \sum_{j=1}^{K}\left\|\boldsymbol{x}_{j}^{\boldsymbol{B}}-\boldsymbol{x}^{\star}\right\|_{2}^{2}-\mathcal{C}_{1}^{2}(\delta)\left(\mathbb{E}\|\boldsymbol{z}[\mathcal{I}]\|_{2}^{2}+\tau^{2}\right) \leq 0\right\}
\end{aligned}
$$

By independence, we can factorize the two probabilities:

$$
\begin{aligned}
& =\mathbb{P}\left\{\left\|\boldsymbol{x}^{\boldsymbol{B}}-\boldsymbol{x}^{\star}\right\|_{2}^{2}-\frac{1}{K} \sum_{j=1}^{K}\left\|\boldsymbol{x}_{j}^{\boldsymbol{B}}-\boldsymbol{x}^{\star}\right\|_{2}^{2} \leq 0\right\} \\
& \cdot \mathbb{P}\left\{\sum_{j=1}^{K}\left\|\boldsymbol{x}_{j}^{\boldsymbol{B}}-\boldsymbol{x}^{\star}\right\|_{2}^{2}-K \mathcal{C}_{1}{ }^{2}(\delta)\left(\mathbb{E}\|\boldsymbol{z}[\mathcal{I}]\|_{2}^{2}+\tau^{2}\right) \leq 0\right\}
\end{aligned}
$$

By Jensens' Inequality, the first term is 1 and

$$
\begin{aligned}
& \mathbb{P}\left\{\left\|\boldsymbol{x}^{\boldsymbol{B}}-\boldsymbol{x}^{\star}\right\|_{2}-\mathcal{C}_{1}(\delta)\left(\left(\mathbb{E}\|\boldsymbol{z}[\mathcal{I}]\|_{2}^{2}\right)^{1 / 2}+\tau\right) \leq 0\right\} \\
& \geq \mathbb{P}\left\{\sum_{j=1}^{K}\left\|\boldsymbol{x}_{j}^{\boldsymbol{B}}-\boldsymbol{x}^{\star}\right\|_{2}^{2}-K \mathcal{C}_{1}{ }^{2}(\delta)\left(\mathbb{E}\|\boldsymbol{z}[\mathcal{I}]\|_{2}^{2}+\tau^{2}\right) \leq 0\right\} .
\end{aligned}
$$


From this procedure, we reduce the error bound for the bagging algorithm to bound the sum of individual errors.

We let random variable $\underline{\boldsymbol{x}}=\left\|\boldsymbol{x}(\mathcal{I})-\boldsymbol{x}^{\star}\right\|_{2}^{2}$, where $\boldsymbol{x}(\mathcal{I})$ is the solution from $\ell_{1}$ minimization on bootstrap samples of size $L: \boldsymbol{x}(\mathcal{I})=\arg \min \|\boldsymbol{x}\|_{1}$ s.t. $\|\boldsymbol{y}[\mathcal{I}]-\boldsymbol{A}[\mathcal{I}]\|_{2}^{2} \leq \epsilon$, where $\mathcal{I}$ denotes a bootstrap sample. All $\boldsymbol{x}_{j}=\left\|\boldsymbol{x}_{j}^{\boldsymbol{B}}-\boldsymbol{x}^{\star}\right\|_{2}^{2}$ are realizations generated i.i.d. from the distribution of $\underline{x}$. We proceed the proof using the following lemma that gives the tail bound of the sum of i.i.d. bounded random variables, and its proof follows a similar procedure as proving Hoeffdings' inequality (details in Appendix VIII-E).

Lemma 18 (Tail bound of the sum of i.i.d. bounded Random variables). Let $Y_{1}, Y_{2}, \ldots, Y_{n}$ be i.i.d. observations of bounded random variable $Y: a \leq Y \leq b$ and the expectation $\mathbb{E} Y$ exists, for any $\epsilon>0$, then

$$
\mathbb{P}\left\{\sum_{i=1}^{n} Y_{i} \geq n \epsilon\right\} \leq \exp \left\{-\frac{2 n(\epsilon-\mathbb{E} Y)^{2}}{(b-a)^{2}}\right\} .
$$

In this case, we consider the lower bound $a$ and the upper bound $b$ of random variable $\underline{x}$. Clearly $\underline{\boldsymbol{x}} \geq 0$, we therefore set $a=0$. The upper bound is obtained from the error bound of $\ell_{1}$ minimization in Theorem 6 . For all $\mathcal{I}$ :

$$
\mathbb{P}\left\{\left\|\boldsymbol{x}(\mathcal{I})-\boldsymbol{x}^{\star}\right\|_{2}^{2}-\mathcal{C}_{1}{ }^{2}(\delta)\|\boldsymbol{z}[\mathcal{I}]\|_{2}^{2} \leq 0\right\}=1,
$$

According to the norm equivalence inequality

$$
\|\boldsymbol{z}[\mathcal{I}]\|_{2}^{2} \leq\left(\sqrt{L}\|\boldsymbol{z}[\mathcal{I}]\|_{\infty}\right)^{2} \leq\left(\sqrt{L}\|\boldsymbol{z}\|_{\infty}\right)^{2}=L\|\boldsymbol{z}\|_{\infty}^{2}
$$

and we set $b=\mathcal{C}_{1}{ }^{2}(\delta) L\|\boldsymbol{z}\|_{\infty}^{2}$.

Now we can apply use (43) to analyze our problem. By 42, the $\epsilon$ in 43 is: $\epsilon=\mathcal{C}_{1}^{2}(\delta)\left(\mathbb{E}\|\boldsymbol{z}[\mathcal{I}]\|_{2}^{2}+\tau^{2}\right)$, then

$$
\mathbb{P}\left\{\sum_{j=1}^{K}\left\|\boldsymbol{x}_{j}-\boldsymbol{x}^{\star}\right\|_{2}^{2}-k \epsilon \geq 0\right\} \leq \exp \left\{-\frac{2 K(\epsilon-\mathbb{E} \underline{\boldsymbol{x}})^{2}}{\mathcal{C}_{1}^{4}(\delta) L^{2}\|\boldsymbol{z}\|_{\infty}^{4}}\right\} .
$$

To simplify the right hand side, we consider: $\mathbb{E} \underline{\boldsymbol{x}}=\mathbb{E} \| \boldsymbol{x}-$ $\boldsymbol{x}^{\star}\left\|_{2}^{2}=\frac{1}{\left|m^{L}\right|} \sum_{\mathcal{I}}\right\| \boldsymbol{x}(\mathcal{I})-\boldsymbol{x}^{\star} \|_{2}^{2}$. From our bound in 44, , it implies that

$$
\mathbb{P}\left\{\frac{1}{\left|m^{L}\right|} \sum_{\mathcal{I}}\left\|\boldsymbol{x}_{\mathcal{I}}-\boldsymbol{x}^{\star}\right\|_{2}^{2} \leq \frac{1}{\left|m^{L}\right|} \sum_{\mathcal{I}} \mathcal{C}_{1}^{2}(\delta)\left\|\boldsymbol{z}_{\mathcal{I}}\right\|_{2}^{2}\right\}=1
$$

which is equivalent to

$$
\begin{aligned}
\mathbb{E}\left\|\boldsymbol{x}(\mathcal{I})-\boldsymbol{x}^{\star}\right\|_{2}^{2} & \leq \frac{1}{\left|m^{L}\right|} \sum_{\mathcal{I}} \mathcal{C}_{1}^{2}(\delta)\|\boldsymbol{z}[\mathcal{I}]\|_{2}^{2} \\
& =\mathbb{E} \mathcal{C}_{1}^{2}(\delta)\left\|\boldsymbol{z}_{\mathcal{I}}\right\|_{2}^{2}=\mathcal{C}_{1}{ }^{2}(\delta) \mathbb{E}\left\|\boldsymbol{z}_{\mathcal{I}}\right\|_{2}^{2}
\end{aligned}
$$

Then we have

$$
\begin{gathered}
\epsilon-\mathbb{E} \underline{\boldsymbol{x}}=\mathcal{C}_{1}{ }^{2}(\delta)\left(\mathbb{E}\|\boldsymbol{z}[\mathcal{I}]\|_{2}^{2}+\tau^{2}\right)-\mathbb{E}\left\|\boldsymbol{x}-\boldsymbol{x}^{\star}\right\|_{2}^{2} \\
\geq \mathcal{C}_{1}^{2}(\delta)\left(\mathbb{E}\|\boldsymbol{z}[\mathcal{I}]\|_{2}^{2}+\tau^{2}\right)-\mathcal{C}_{1}{ }^{2}(\delta) \mathbb{E}\left\|\boldsymbol{z}_{\mathcal{I}}\right\|_{2}^{2}=\mathcal{C}_{1}{ }^{2}(\delta) \tau^{2}
\end{gathered}
$$

The right hand side of 46 is upper bounded by $\exp \left\{-\frac{2 K \tau^{4}}{L^{2}\|\boldsymbol{z}\|_{\infty}^{4}}\right\}$.
E. Proof of Theorem 15 performance bound of bagging for general sparse signal recovery

In this section, we are working with the case when the true solution $\boldsymbol{x}^{\star}$ is a general sparse signal, which sparsity level may exceed $s$ and the $s$-sparse approximation error is no longer necessarily zero. Let $\epsilon_{s}$ denote the sparse approximation error $\epsilon_{s}=\mathcal{C}_{0}(\delta) s^{-1 / 2}\|\boldsymbol{e}\|_{1}$, we consider the following:

$$
\begin{aligned}
& \mathbb{P}\left\{\left\|\boldsymbol{x}^{\boldsymbol{B}}-\boldsymbol{x}^{\star}\right\|_{2}-\left(\epsilon_{s}+\mathcal{C}_{1}(\delta)\left(\sqrt{\frac{L}{m}}\|\boldsymbol{z}\|_{2}+\tau\right)\right) \leq 0\right\} \\
& =\mathbb{P}\left\{\left\|\boldsymbol{x}^{\boldsymbol{B}}-\boldsymbol{x}^{\star}\right\|_{2}^{2}-\left(\epsilon_{s}+\mathcal{C}_{1}(\delta)\left(\sqrt{\frac{L}{m}}\|\boldsymbol{z}\|_{2}+\tau\right)\right)^{2} \leq 0\right\} \\
& \geq \mathbb{P}\left\{\left\|\boldsymbol{x}^{\boldsymbol{B}}-\boldsymbol{x}^{\star}\right\|_{2}^{2}-\left(\left(\epsilon_{s}+\mathcal{C}_{1}(\delta) \sqrt{\frac{L}{m}}\|\boldsymbol{z}\|_{2}\right)^{2}+\mathcal{C}_{1}^{2}(\delta) \tau^{2}\right) \leq 0\right\}
\end{aligned}
$$

We let $\left.\epsilon^{\prime}=\left(\epsilon_{s}+\mathcal{C}_{1}(\delta) \sqrt{\frac{L}{m}}\|\boldsymbol{z}\|_{2}\right)^{2}+\mathcal{C}_{1}{ }^{2}(\delta) \tau^{2}\right)$ and we consider using the averages of the errors $\frac{1}{K} \sum_{j=1}^{K}\left\|\boldsymbol{x}_{j}^{\boldsymbol{B}}-\boldsymbol{x}^{\star}\right\|_{2}^{2}$ as an intermediate term. Repeat the same proving technique as we did in 42, we have

$$
\mathbb{P}\left\{\left\|\boldsymbol{x}^{\boldsymbol{B}}-\boldsymbol{x}^{\star}\right\|_{2}^{2}-\epsilon^{\prime}\right\} \geq \mathbb{P}\left\{\sum_{j=1}^{K}\left\|\boldsymbol{x}_{j}^{\boldsymbol{B}}-\boldsymbol{x}^{\star}\right\|_{2}^{2}-K \epsilon^{\prime} \leq 0\right\} .
$$

According to Lemma 18, we have:

$$
\mathbb{P}\left\{\sum_{j=1}^{K}\left\|\boldsymbol{x}_{j}^{\boldsymbol{B}}-\boldsymbol{x}^{\star}\right\|_{2}^{2}-K \epsilon^{\prime} \geq 0\right\} \leq \exp \left\{-\frac{2 K\left(\epsilon^{\prime}-\mathbb{E} \underline{\boldsymbol{x}}\right)^{2}}{\left(b^{\prime}-a^{\prime}\right)^{2}}\right\} .
$$

Here $a^{\prime}=0$, and $b^{\prime}=\left(\epsilon_{s}+\mathcal{C}_{1}(\delta) \sqrt{L}\|\boldsymbol{z}\|_{\infty}\right)^{2}$. The lower bound $a^{\prime}$ is set to zero since $\underline{\boldsymbol{x}}$ is non negative and the upper bound $b^{\prime}$ is obtained using Theorem 6 and plug in the upper bound of the noise power as derived in (45).

We consider the term $\epsilon^{\prime}-\mathbb{E} \underline{\boldsymbol{x}}=\left(\mathcal{C}_{0}(\delta) s^{-1 / 2}\|\boldsymbol{e}\|_{1}+\right.$ $\left.\mathcal{C}_{1}(\delta) \sqrt{\frac{L}{m}}\|\boldsymbol{z}\|_{2}\right)^{2}+\mathcal{C}_{1}^{2}(\delta) \tau^{2}-\mathbb{E}\left\|\boldsymbol{x}-\boldsymbol{x}^{\star}\right\|_{2}^{2}$. We upper bound the expected value of $\underline{x}$ in the same approach as in 47 . From Theorem 6 , for all $\mathcal{I}$ :

$$
\mathbb{P}\left\{\left\|\boldsymbol{x}(\mathcal{I})-\boldsymbol{x}^{\star}\right\|_{2}^{2} \leq\left(\epsilon_{s}+\mathcal{C}_{1}(\delta)\|\boldsymbol{z}[\mathcal{I}]\|_{2}\right)^{2}\right\}=1 .
$$

Therefore,

$$
\mathbb{P}\left\{\mathbb{E}\left\|\boldsymbol{x}[\mathcal{I}]-\boldsymbol{x}^{\star}\right\|_{2}^{2} \leq \mathbb{E}\left(\epsilon_{s}+\mathcal{C}_{1}(\delta)\|\boldsymbol{z}[\mathcal{I}]\|_{2}\right)^{2}\right\}=1 .
$$

Because $f(x)=x^{2}$ is a convex function, and therefore by Jensens' inequality, we have:

$$
\left(\mathbb{E}\|\boldsymbol{z}[\mathcal{I}]\|_{2}\right)^{2} \leq \mathbb{E}\|\boldsymbol{z}[\mathcal{I}]\|_{2}^{2} .
$$

Because square root $x^{1 / 2}$ is a increasing function with $x$, therefore taking square root preserves the sign of inequality:

$$
\mathbb{E}\|\boldsymbol{z}[\mathcal{I}]\|_{2} \leq\left(\mathbb{E}\|\boldsymbol{z}[\mathcal{I}]\|_{2}^{2}\right)^{1 / 2} .
$$

Then from 50, we have:

$$
\begin{aligned}
& \mathbb{E}\left\|\boldsymbol{x}[\mathcal{I}]-\boldsymbol{x}^{\star}\right\|_{2}^{2} \leq \mathbb{E}\left(\epsilon_{s}+\mathcal{C}_{1}(\delta)\|\boldsymbol{z}[\mathcal{I}]\|_{2}\right)^{2} \\
& \quad=\epsilon_{s}^{2}+\mathcal{C}_{1}^{2}(\delta) \mathbb{E}\|\boldsymbol{z}[\mathcal{I}]\|_{2}^{2}+2 \epsilon_{s} \mathcal{C}_{1}(\delta) \mathbb{E}\|\boldsymbol{z}[\mathcal{I}]\|_{2}
\end{aligned}
$$

(by (51))

$$
\begin{aligned}
& \leq \epsilon_{s}^{2}+\mathcal{C}_{1}^{2}(\delta) \mathbb{E}\|\boldsymbol{z}[\mathcal{I}]\|_{2}^{2}+2 \epsilon_{s} \mathcal{C}_{1}(\delta)\left(\mathbb{E}\left\|\boldsymbol{z}_{\mathcal{I}}\right\|_{2}^{2}\right)^{1 / 2} \\
& =\left(\epsilon_{s}+\mathcal{C}_{1}(\delta)\left(\mathbb{E}\|\boldsymbol{z}[\mathcal{I}]\|_{2}^{2}\right)^{1 / 2}\right)^{2}
\end{aligned}
$$


From previous result, we have $\left(\mathbb{E}\|\boldsymbol{z}[\mathcal{I}]\|_{2}^{2}\right)^{1 / 2}=\sqrt{\frac{L}{m}}\|\boldsymbol{z}\|_{2}$ and therefore $\epsilon^{\prime}=\left(\epsilon_{s}+\mathcal{C}_{1}(\delta)\left(\mathbb{E}\|\boldsymbol{z}[\mathcal{I}]\|_{2}^{2}\right)^{1 / 2}\right)^{2}+\mathcal{C}_{1}^{2}(\delta) \tau^{2}$. Then we can bound the term $\epsilon^{\prime}-\mathbb{E}\left\|\boldsymbol{x}-\boldsymbol{x}^{\star}\right\|_{2}^{2}$ :

$$
\begin{aligned}
& \epsilon^{\prime}-\mathbb{E}\left\|\boldsymbol{x}-\boldsymbol{x}^{\star}\right\|_{2}^{2} \\
= & \left(\epsilon_{s}+\mathcal{C}_{1}(\delta)\left(\mathbb{E}\|\boldsymbol{z}[\mathcal{I}]\|_{2}^{2}\right)^{1 / 2}\right)^{2}+\mathcal{C}_{1}{ }^{2}(\delta) \tau^{2}-\mathbb{E}\left\|\boldsymbol{x}-\boldsymbol{x}^{\star}\right\|_{2}^{2} \\
\geq & \left(\left(\epsilon_{s}+\mathcal{C}_{1}(\delta)\left(\mathbb{E}\|\boldsymbol{z}[\mathcal{I}]\|_{2}^{2}\right)^{1 / 2}\right)^{2}+\mathcal{C}_{1}{ }^{2}(\delta) \tau^{2}\right. \\
& -\left(\epsilon_{s}+\mathcal{C}_{1}(\delta)\left(\mathbb{E}\|\boldsymbol{z}[\mathcal{I}]\|_{2}^{2}\right)^{1 / 2}\right)^{2}=\mathcal{C}_{1}(\delta)^{2} \tau^{2} .
\end{aligned}
$$

The bound in 49) can be upper bounded by

$$
\mathbb{P}\left\{\sum_{j=1}^{K}\left\|\boldsymbol{x}_{j}^{B}-\boldsymbol{x}^{\star}\right\|_{2}^{2}-K \epsilon^{\prime} \geq 0\right\} \leq \exp \left\{-\frac{2 K \mathcal{C}_{1}^{4}(\delta) \tau^{4}}{\left(b^{\prime}\right)^{2}}\right\} .
$$

where $b^{\prime}=\left(\mathcal{C}_{0}(\delta) s^{-1 / 2}\|\boldsymbol{e}\|_{1}+\mathcal{C}_{1}(\delta) \sqrt{L}\|\boldsymbol{z}\|_{\infty}\right)^{2}$.

\section{F. Sufficient condition: Theorem 16 from Sample Complexity for gaussian and bernoulli random matrices}

We would like to connect the BRIP constant of $A^{J}$ to RIP constants all submatrices $\boldsymbol{A}\left[\mathcal{I}_{j}\right]$ s. First, we consider using $V$ to represent the number of distinct measurements of bootstrapping samples of size $L$, and $0 \leq L \leq m$. Pick $d<L$ being the smallest number of distinct samples that we would like to have hold with probability at least $1-\alpha$, which is

$$
\mathbb{P}(V \geq d) \geq 1-\alpha .
$$

The relationship of $d, L, m$ and the $\alpha$ given the rest variables can be found in Appendix VIII-D in (69).

Let $V_{1}, V_{2}, \ldots, V_{k}$ count the number of distinct measurements of all sub-measurements $\boldsymbol{y}\left[\mathcal{I}_{1}\right], \boldsymbol{y}\left[\mathcal{I}_{2}\right], \ldots, \boldsymbol{y}\left[\mathcal{I}_{K}\right]$. Because of the bootstrap procedure, $V_{i}$ s are i.i.d. distributed as random variable $V$. Consider the probability that all the $V_{i} \geq d$, we have:

$$
\begin{aligned}
\mathbb{P}\left\{\forall j V_{j} \geq d\right\}=\mathbb{P}\left\{\bigcap_{j=1}^{K}\left\{V_{j} \geq d\right\}\right\} & =\prod_{j=1}^{K} \mathbb{P}\left\{V_{j} \geq d\right\} \\
& \geq(1-\alpha)^{K} .
\end{aligned}
$$

We would like to calculate the BRIP constant of $A^{J}=$ $\operatorname{diag}\left(\boldsymbol{A}\left[\mathcal{I}_{1}\right], \boldsymbol{A}\left[\mathcal{I}_{2}\right], \ldots, \boldsymbol{A}\left[\mathcal{I}_{K}\right]\right)$. To simplify the process, we first consider the same certainty level $\mu_{J}$ and lower bound of number of distinct samples $d$ for each for the standard RIP constant for each sub-marix $\boldsymbol{A}\left[\mathcal{I}_{j}\right]$. Entries of the distinct rows of each sub-matrix come from Gaussian distribution. According to Theorem 8 , if we have enough distinct measurements $d \geq \beta \delta^{-2}\left(2 s \ln (n / 2 s)+\ln \left(\mu_{J}^{-1}\right)\right)$, then for $\mu_{J}, d$, all $j=1,2, \ldots, K$

$$
\mathbb{P}\left\{\delta_{2 s}\left(\boldsymbol{A}\left[\mathcal{I}_{j}\right]\right) \leq \delta \mid V_{j} \geq d\right\} \geq 1-\mu_{J},
$$

Note that, here the RIP constant of $\boldsymbol{A}\left[\mathcal{I}_{j}\right]$ considers the RIP constant on distinct rows of $\boldsymbol{A}\left[\mathcal{I}_{j}\right]$.

Now we consider the BRIP constant of $\boldsymbol{A}^{J}$, given the condition that all sub-matrices has at least $d$ distinct measurements. According to (11) in Proposition 11, we have

$$
\begin{aligned}
& \mathbb{P}\left\{\delta_{2 s \mid \mathcal{B}}\left(\boldsymbol{A}^{\boldsymbol{J}}\right)=\max _{j} \delta_{2 s}\left(\boldsymbol{A}\left[\mathcal{I}_{j}\right]\right) \leq \delta \mid \forall j V_{j} \geq d\right\} \\
& =\mathbb{P}\left\{\forall j=1,2, \ldots, m: \delta_{2 s}\left(\boldsymbol{A}\left[\mathcal{I}_{j}\right]\right) \leq \delta \mid V_{j} \geq d\right\} \\
& =1-\mathbb{P}\left\{\exists j=1,2, \ldots, m: \delta_{2 s}\left(\boldsymbol{A}\left[\mathcal{I}_{j}\right]\right)>\delta \mid V_{j} \geq d\right\}
\end{aligned}
$$

Note that although $\boldsymbol{A}\left[\mathcal{I}_{j}\right]$ are not mutually independent, we can employ union bound:

$$
\begin{aligned}
& \geq 1-\sum_{i=1}^{K} \mathbb{P}\left\{\delta_{2 s}\left(\boldsymbol{A}\left[\mathcal{I}_{j}\right]\right)>\delta \mid V_{j} \geq d\right\} \\
& \geq 1-K \mu_{J} .
\end{aligned}
$$

Finally, we consider the BRIP constant of $A^{J}$

$$
\begin{aligned}
& \mathbb{P}\left\{\delta_{2 s \mid \mathcal{B}}\left(\boldsymbol{A}^{\boldsymbol{J}}\right) \leq \delta\right\}=\mathbb{P}\left\{\max _{j} \delta_{2 s}\left(\boldsymbol{A}\left[\mathcal{I}_{j}\right]\right) \leq \delta\right\} \\
& =\mathbb{P}\left\{\max _{j} \delta_{2 s}\left(\boldsymbol{A}\left[\mathcal{I}_{j}\right]\right) \leq \delta \mid \forall j V_{j} \geq d\right\} \mathbb{P}\left\{\forall j V_{j} \geq d\right\} \\
& +\mathbb{P}\left\{\max _{j} \delta_{2 s}\left(\boldsymbol{A}\left[\mathcal{I}_{j}\right]\right) \leq \delta \mid \exists j V_{j}<d\right\} \mathbb{P}\left\{\exists V_{j}<d\right\} \\
& \geq \mathbb{P}\left\{\max _{j} \delta_{2 s}\left(\boldsymbol{A}\left[\mathcal{I}_{j}\right]\right) \leq \delta \mid \forall j V_{j} \geq d\right\} \mathbb{P}\left\{\forall j V_{j} \geq d\right\}
\end{aligned}
$$

We here drop the second term to get a lower bound. According to 54): $\mathbb{P}\left\{\exists V_{i}<d\right\} \leq 1-(1-\alpha)^{K}$, which is fairly small when $\alpha$ is small. The choice of $\alpha$ is preferred to be small in practice and the bound is a good for practical proposes since it is tighter when $\alpha$ is smaller. Then by (54), we have:

$$
\begin{aligned}
& \mathbb{P}\left\{\max _{j} \delta_{2 s}\left(\boldsymbol{A}\left[\mathcal{I}_{j}\right]\right) \leq \delta\right\} \\
& \geq \mathbb{P}\left\{\max _{j} \delta_{2 s}\left(\boldsymbol{A}\left[\mathcal{I}_{j}\right]\right) \leq \delta \mid \forall j V_{j} \geq d\right\} \mathbb{P}\left\{\forall j V_{j} \geq d\right\} \\
& \geq\left(1-K \mu_{J}\right)(1-\alpha)^{K} .
\end{aligned}
$$

To simplify the bound in (57), we would like to achieve

$$
\left(1-K \mu_{J}\right)(1-\alpha)^{K} \geq 1-\mu, \text { for some } \mu \in[0,1] .
$$

Namely,

$$
\begin{aligned}
& \left(1-K \mu_{J}\right)(1-\alpha)^{K} \geq 1-\mu \\
\Longleftrightarrow & \left(1-K \mu_{J}\right) \geq \frac{1-\mu}{(1-\alpha)^{K}} \\
\Longleftrightarrow & \mu_{J} \leq \frac{1}{K}-\frac{1-\mu}{K(1-\alpha)^{K}}=\frac{(1-\alpha)^{K}-(1-\mu)}{K(1-\alpha)^{K}} .
\end{aligned}
$$

According to Theorem 8, (58) can be achieved if

$$
\begin{aligned}
d & >\beta \delta^{-2}\left(2 s \ln (n / 2 s)+\ln \left(\mu_{J}^{-1}\right)\right) \\
& \geq \beta \delta^{-2}\left(2 s \ln (n / 2 s)-\ln \left(\frac{(1-\alpha)^{K}-(1-\mu)}{K(1-\alpha)^{K}}\right)\right. \\
& =\beta \delta^{-2}\left(2 s \ln (n / 2 s)+\ln K+\ln \left(\frac{(1-\alpha)^{K}}{(1-\alpha)^{K}-(1-\mu)}\right)\right) .
\end{aligned}
$$

Replace $\delta_{2 s}\left(\mu_{J}, d(\alpha, m, L)\right)$ by its upper bound $\sqrt{2}-1$ and therefore the sample complexity is $\mathcal{O}(2 s \ln (n / 2 s)+\ln K+$ $\left.\ln \left(\frac{(1-\alpha)^{K}}{(1-\alpha)^{K}-(1-\mu)}\right)\right)$ and the constant depends on $\mu$ and $\alpha$. Both the last two terms of $60 p: \ln K+\ln \left(\frac{(1-\alpha)^{K}}{(1-\alpha)^{K}-(1-\mu)}\right)$ are introduced by the uncertainty introduced by the bootstrap procedure and they are all non-decreasing with respect to $K$. This theorem also matches the RIP condition that increasing $K$ by adding extra multi-sets $\mathcal{I}_{s}$, the RIP constant will guarantee to be non-decreasing.

Note that there are some limitations of this theorem. The prove follows standard RIP condition for sparse recovery, however, the range that RIP condition guarantees are not wide 
enough: in this case, the worst case performance is limited by the worst $\boldsymbol{A}\left[\mathcal{I}_{i}\right]$. As a result the probably of success being guaranteed in 57 has $(1-\alpha)^{K}$, which will vanish fast if $K$ is large and then the bound becomes quite loose. Also, there is an implicit condition while proving: to guarantee all the probabilities to be between zero and one, while $K>1$, equation (58) implicitly implies that $\mu \geq \alpha$. This means that, the certainty of the performance of the algorithm is limited by the certainty level of the minimum number of distinct measurements across each column in the measurement matrix. This implicit assumption makes sense however it is a bit conservative to estimate performances in practice.

\section{Simulations}

In this section, we perform sparse recovery on simulated data to study the performance of our algorithm. In our experiment, all entries of $\boldsymbol{A} \in \mathbb{R}^{m \times n}$ are i.i.d. samples from the standard normal distribution $\mathcal{N}(0,1)$. This simulation setting is the same as the one in the analysis part by multiplying a normalization factor $\frac{1}{m}$. The signal dimension $n=200$ and various numbers of measurements from 50 to 2000 are explored. For the ground truth signals, their sparsity levels are $s=50$, and the nonzeros entries are sampled from the standard gaussian with their locations being generated uniformly at random. For the noise processes $\boldsymbol{z}$, which entries are sampled i.i.d. from $\mathcal{N}\left(0, \sigma^{2}\right)$, with variance $\sigma^{2}=10^{-\mathrm{SNR} / 10}\|\boldsymbol{A} \boldsymbol{x}\|_{2}^{2}$, where SNR represents the Signal to Noise Ratio. In our experiment, we study three different ratios: $\mathrm{SNR}=0,1$ and $2 \mathrm{~dB}$.

We use the ADMM implementation of Block (Group) LASSO [21] to solve the unconstraint form of $\mathbf{J}_{12}^{\boldsymbol{J}_{2}}$, in which the parameter $\lambda^{(k, L)}$ balances the least squares fit and the joint sparsity penalty:

$$
\min _{\boldsymbol{X}} \lambda^{(K, L)}\|\boldsymbol{X}\|_{1,2}+\frac{1}{2} \sum_{j=1}^{K}\left\|\boldsymbol{y}\left[\mathcal{I}_{j}\right]-\boldsymbol{A}\left[\mathcal{I}_{j}\right] \boldsymbol{x}_{j}\right\|_{2}^{2} .
$$

The same solver is used to solve $\ell_{1}$ minimization with $K=1$ for a fair comparison with all other algorithms.

We study how the number of estimates $K$ as well as the bootstrapping ratio $L / m$ affects the result. In our experiment, we take $K=30,50,100$, while the bootstrap ratio $L / m$ varies from 0.1 to 1 . We report the Signal to Noise Ratio (SNR) as the error measure for recovery : SNR $=10 \log _{10}\left\|\boldsymbol{x}-\boldsymbol{x}^{\star}\right\|_{2}^{2} /\left\|\boldsymbol{x}^{\star}\right\|_{2}^{2}$ averaged over 20 independent trials. For all algorithms, we evaluate $\lambda^{(K, L)}$ at different values from .01 to 200 and then select optimal values that gives the maximum averaged SNR over all trials.

\section{A. Performance of JOBS, Bagging, Bolasso and $\ell_{1}$ minimiza- tion}

Beside JOBS, Bagging and Bolasso with the same parameters $K, L$ and $\ell_{1}$ minimization are studied. The result are plotted in Figure 2 and Figure 3 . The colored curves shows the cases with various number of estimates $K$. The grey circle highlights the best performance and the grey area highlights the optimal bootstrap ratio $L / m$. In those figures, for each condition with a choice of $K, L$, the information available to JOBS, Bagging and Bolasso algorithms is identical, and $\ell_{1}$
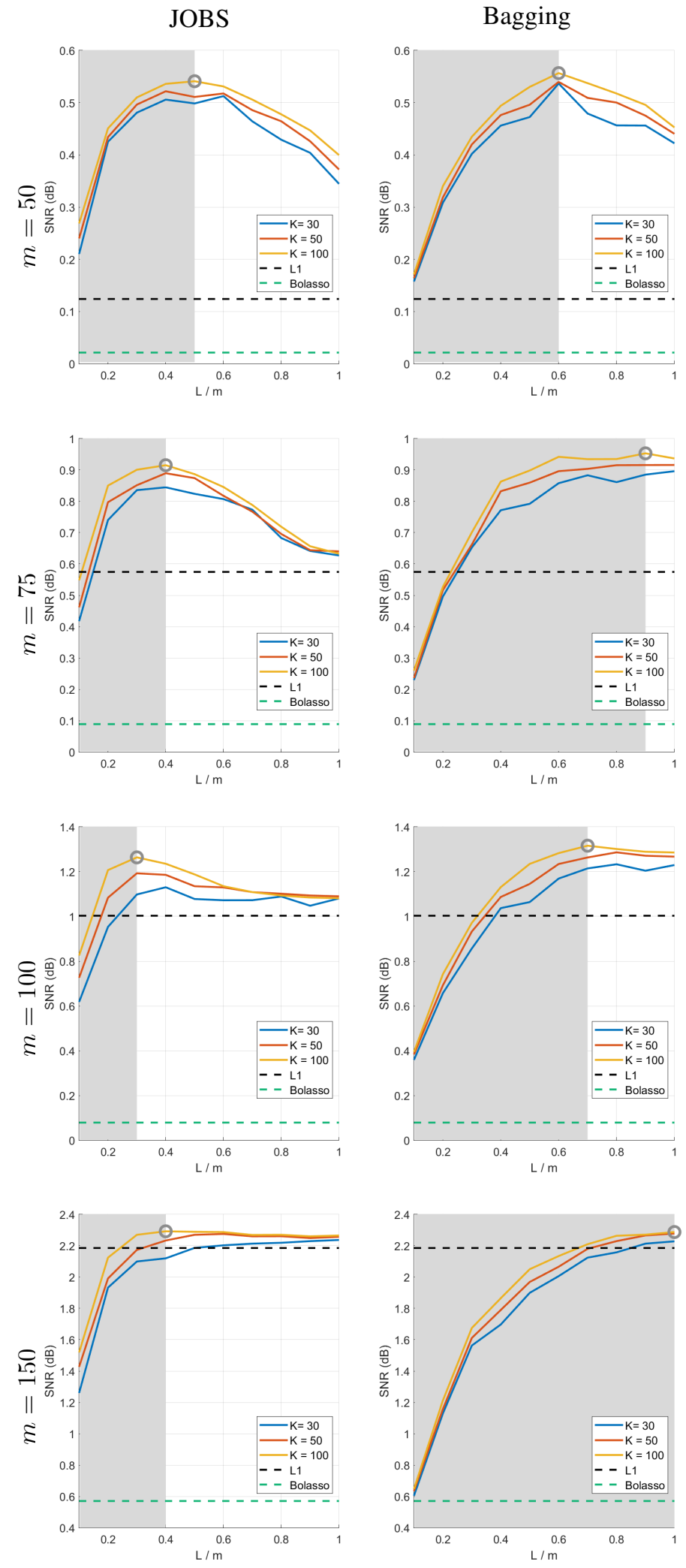

Fig. 2. Performance curves for JOBS, Bagging and Bolasso with different $L, K$ as well as $\ell_{1}$ minimization. SNR $=0 \mathrm{~dB}$ and the number of measurements $m=50,75,100,150$ from top to bottom. The grey circle highlights the peaks of JOBS, Bagging and Bolasso and the grey area highlights the bootstrap ratio at the peak point. JOBS requires smaller $L / m$ than Bagging to achieve peak performance. JOBS and Bagging outperform $\ell_{1}$ minimization and Bolasso when $m$ is small. 

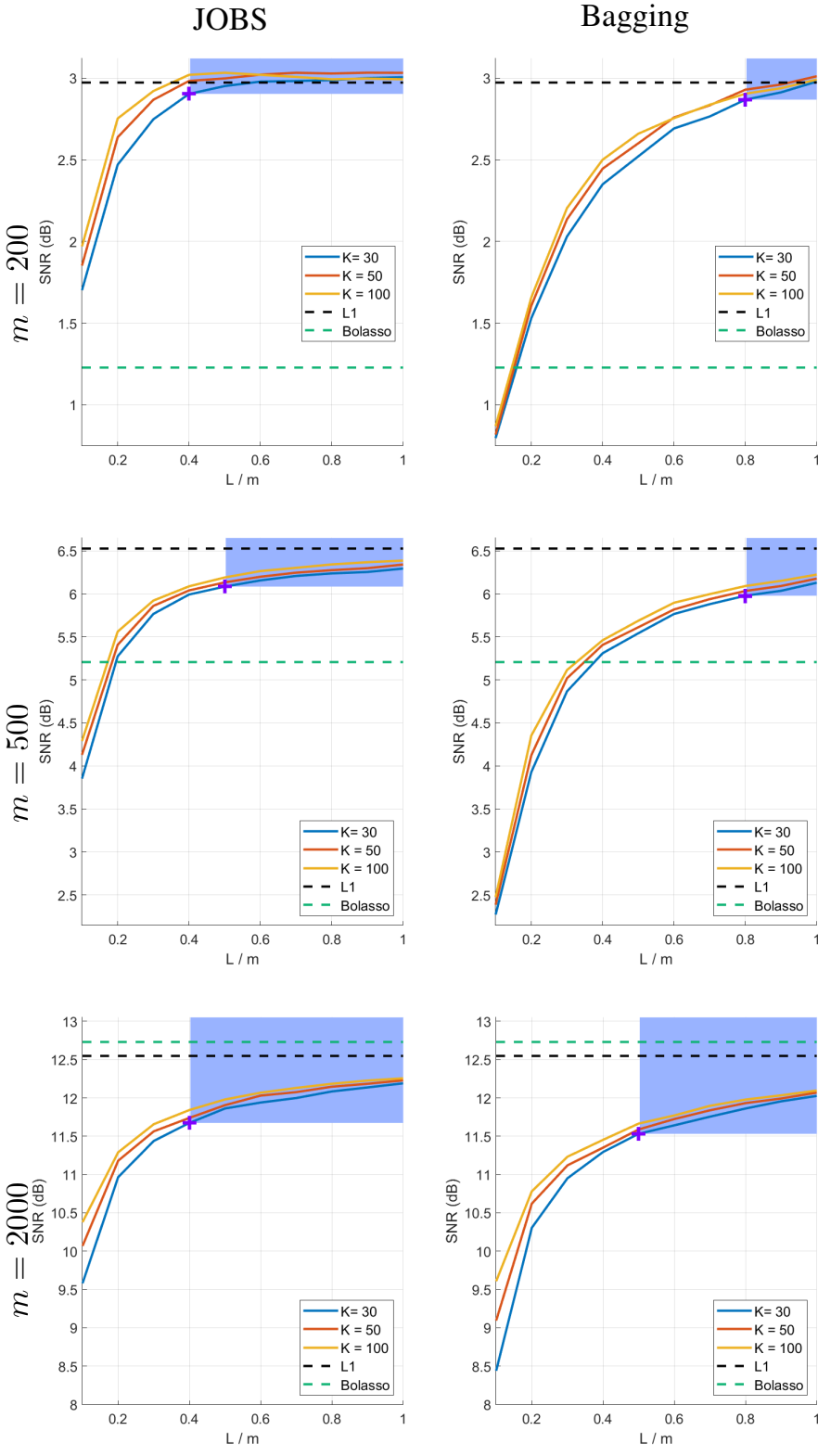

Fig. 3. Performance curves for JOBS, Bagging and Bolasso with different $L, K$ as well as $\ell_{1}$ minimization. $\mathrm{SNR}=0 \mathrm{~dB}$ and the number of measurements $m=200,500,2000$ from top to bottom. The purple highlighted area is where at least $95 \%$ of the best performance achieved and within which the minimum $K \times L$ is illustrated by purple cross. JOBS requires smaller $L / m$ than Bagging to achieve acceptable performance.

minimization always access to all $m$ measurements. We plot the performance of JOBS, Bagging with various $L / m$ ratio and $K$. The performance of $\ell_{1}$ minimization is depicted with the black dashed lines, while the best Bolasso performance is plotted using light green dashed lines.

From Figure 2, we see that when $m$ is small, JOBS can outperform $\ell_{1}$ minimization. As $m$ decreases, the margin increases. It is rather surprising that with a low number of measurements ( $m$ is between $s$ to $3 s: 50-150$ ), and with very small $L$ and $K$ ( $L$ about only $30 \%-40 \%$ of the entire set of measurements and $K$ at 30), our algorithm is already quite robust and outperforms all other algorithms with the same $(K, L)$ parameters used. Although in terms of the best performance limit, JOBS and Bagging are similar, Bagging requires $L$ to be around $60 \%-90 \%$ of the entire set of measurements to achieve comparable performance as JOBS. The correct prior information with the row sparsity on multiple estimates may especially show its advantage while the amount of information is limited. However, when the level measurement is high enough, bootstrapping loses its advantages and $\ell_{1}$ becomes the preferred strategy.

Figure 3 shows the performance with on large number of measurements $(m=200,500,2000)$, revealing that JOBS requires a much smaller $L$ to a comparable performance to Bagging. The purple highlighted area is where at least $95 \%$ of the best performance achieved and within which the minimum $K \times L$ is illustrated by purple cross. The purple region are larger and the locations of purple crosses in various $m$ are much further left for our algorithm compared to the ones in Bagging. This implies that although the local maxima for Bagging and JOBS are similar, much smaller $K L$ are required to obtain an acceptable performance. The bootstrapping ratio $L / m$ is $40 \%-50 \%$ for JOBS, $50 \%-80 \%$ for Bagging and $K=30$ for both algorithms to achieve at least $95 \%$ of the best performance for each algorithm. The subsampling variation that we will illustrate in Section VI-B and the result in Figure 6 has an similar advantage. This result is quite promising for large number of measurements. Especially in the streaming setting where utilizing all data at once in a batch algorithm like $\ell_{1}$ minimization is not applicable. When the process is stationary, employing our methods to enforce joint sparsity on multiple local windows boosts performances and the recovery is reasonable with a smaller amount of data.

Figure $4 \mathrm{a}$. Figure $4 \mathrm{~b}$ and Figure $4 \mathrm{c}$ depict the best performance for various schemes: JOBS, Bagging, Bolasso and $\ell_{1}$ minimization with SNR values at $0,1,2 \mathrm{~dB}$ respectively. For the first three algorithms, the peak values are found among different choices of parameters $K$ and $L$ that we explored. We see that when the number of measurements $m$ is low, JOBS and Bagging outperform $\ell_{1}$ minimization. The larger the noise level (lower SNR), the larger the margin. Although the performance limits of JOBS and Bagging are very similar, Figure 2 shows that JOBS achieves comparable performance to Bagging with significantly smaller $L, K$ values. JOBS and Bagging tend to converge to $\ell_{1}$ minimization as $m$ increases. Bolasso only performs similarly to other algorithms for a large $m$ and slightly outperforms all other algorithms when $m=2000$.

\section{B. Subsampling Variation to Ensure Distinct Samples}

Random sampling with replacement (bootstrapping) likely creates duplicates within the samples. Although it simplifies the analysis, in practice, duplicate information does not add much value. Therefore, in this simulation, we conduct a more practical variation of JOBS scheme. To ensure the distinctness within each sample, each time we conduct subsampling, instead of bootstrapping: for each bootstrap sample $\mathcal{I}_{j}, L$ distinct samples are generated by random sampling without replacement from $m$ measurements. There are a few differences compared to the previous bootstrapping scheme: (i) For each subset $\mathcal{I}_{j}$, the information contained for this subsampling variation will 


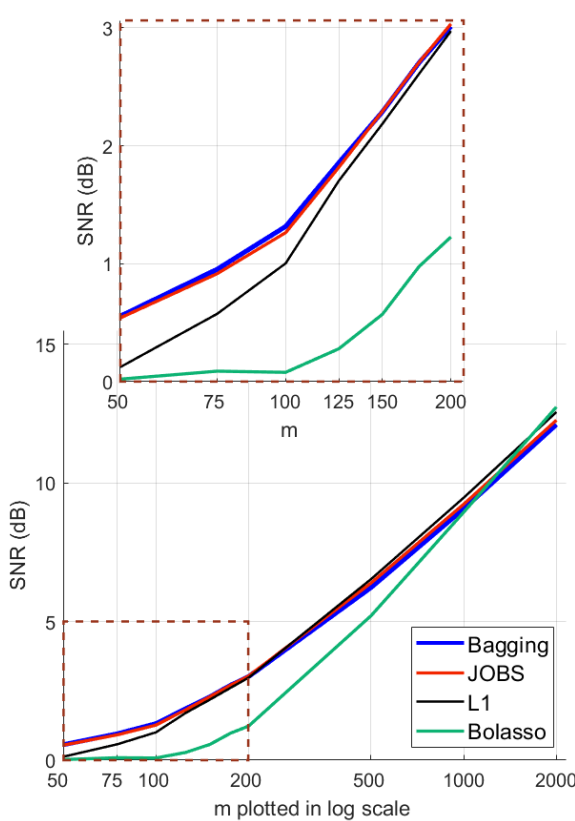

(a) $\mathrm{SNR}=0 \mathrm{~dB}$

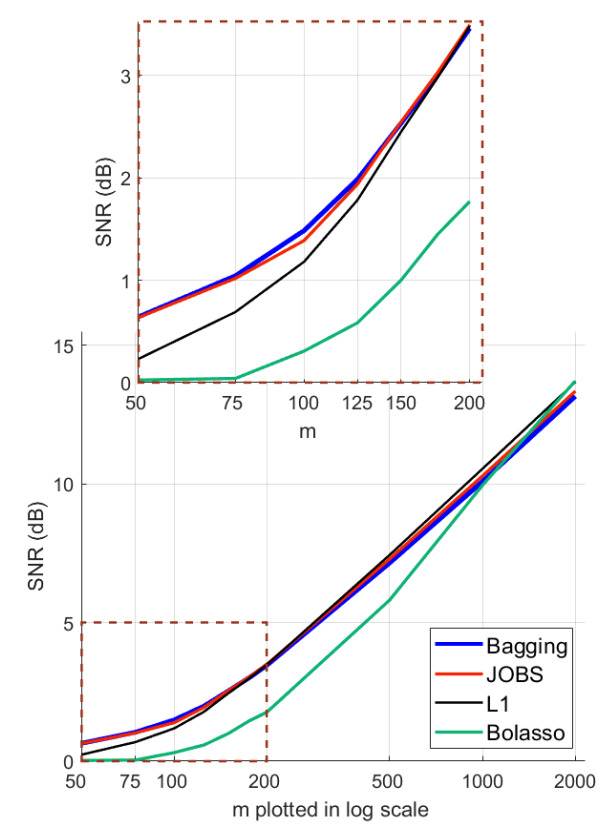

(b) $\mathrm{SNR}=1 \mathrm{~dB}$

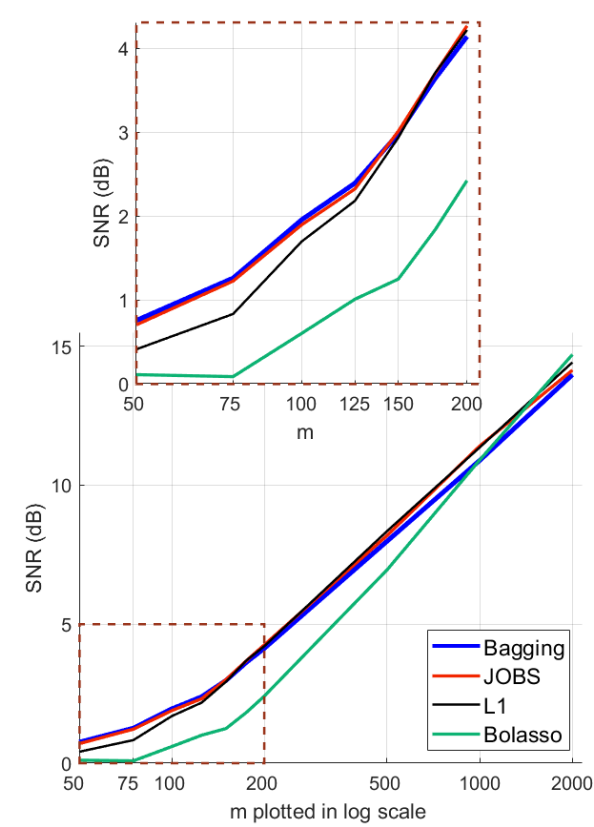

(c) $\mathrm{SNR}=2 \mathrm{~dB}$

Fig. 4. The best performance among all choices of $L$ and $K$ with various number of measurements for JOBS, Bagging, Bolasso and $\ell_{1}$ minimization. While $m$ is small, and lower SNR, the margin between JOBS and $\ell_{1}$ minimization is larger (zoomed-in figures on the top row).

be at least as much as the original scheme. (ii) When the subsampling ratio $L / m=1$, both the subsampling variation of JOBS and Bagging coincides with MMV version of $\ell_{1}$ minimization, and therefore in this case, they all behave the same as $\ell_{1}$ minimization. The original bootstrapping scheme, $L$ is required to be a much larger number than $m$ to observe all $m$ samples.

Similarly to the previous section, we study how the number of estimates $K$ as well as the subsampling ratio $L / m$ affects the result. The variation is also adopted in Bagging and Bolasso. The subsampling version of Bagging is the stochastic approximation of Subagging estimator (short for Subsampling Aggregating) [34], [35]. Here, for simplicity of the terms, we refer all methods by their original names. All the experimental settings are the same as the previous one except the bootstrapping resampling scheme is replaced by subsampling for each subset $\mathcal{I}_{j}$.

Figure 5 depicts the performances of three different algorithms with the same parameters $K, L$ settings. Similar to the case in Figure 2, we see that both JOBS and Bagging outperforms $\ell_{1}$ minimization and JOBS achieves the best performance with smaller $L$ than Bagging. Since subsampling potentially contain more information than bootstrap, it also reduces the length of the subsets $L$ for the best performance. For JOBS, the best subsampling ratio $L / m$ at which the peak value is achieved reduces to $20 \%-40 \%$ for small $m$ (ranging from $50-150$ ), and for Bagging, the optimal subsampling ratio becomes $50 \%-70 \%$. Figure 6 shows the experiments on large number of measurements $(m=200,500,2000)$ with subsampling variation of JOBS, Bagging and Bolasso. The subsampling ratio $L / m$ is $30 \%-50 \%$ for JOBS and $50 \%-70 \%$ for Bagging to achieve at least $95 \%$ of the best performance, which reaches $\ell_{1}$ minimization for the subsampling variation.

Figure 7 depicts the best performance for four different recovery scheme: JOBS, Bagging, Bolasso, all in subsampling variations, and $\ell_{1}$ minimization with SNR values at 0,1 and 2 $\mathrm{dB}$. Similar to Figure 4 when the number of measurements $m$ is low, Bagging and our algorithms outperforms $\ell_{1}$ minimization. The larger the noise level (lower SNR), the larger the margin. As before, Bolasso only outperforms all other three algorithms when the number of measurements is large. While $L=m$, JOBS and Bagging coincide with the $\ell_{1}$ minimization. The optimal values are not that different from the ones in the original bootstrap version in Figure 4 for the same SNR, especially when $m$ are small. While $m$ is large, the original JOBS and Bagging would need the bootstrap ratio to go above 1 to achieve the same result as $\ell_{1}$ minimization and those experiments are not included in this study. We conjecture that the best performance are similar between the original bootstrap scheme and the subsampling variation given the same $m$ with various $K, L$.

With the same $L, K$, the subsampling variation in general gives better performance than bootstrap because more information is likely to be selected. There are two evidences: $(i)$ While $m$ is small, the optimal subsampling ratios $L / m$ for subsampling variations (in Figure 5p are smaller than the optimal bootstrap ratios (in Figure 2) for both JOBS and Bagging since the grey and white boundaries are further left in subsampling variations. (ii) While $m$ is moderate or large, the original JOBS and Bagging start losing advantage to $\ell_{1}$ minimization whereas for the subsampling variations, JOBS and Bagging both approach to $\ell_{1}$ minimization with reasonably small $L / m$ and $K$. Good choices of these two parameters are highlighted in the purple regions in Figure 6 
JOBS
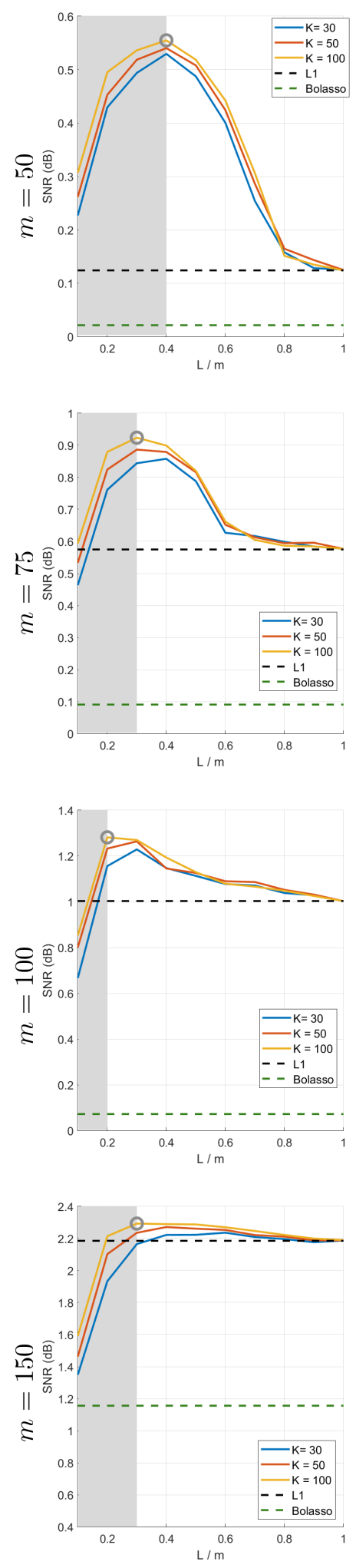

Bagging
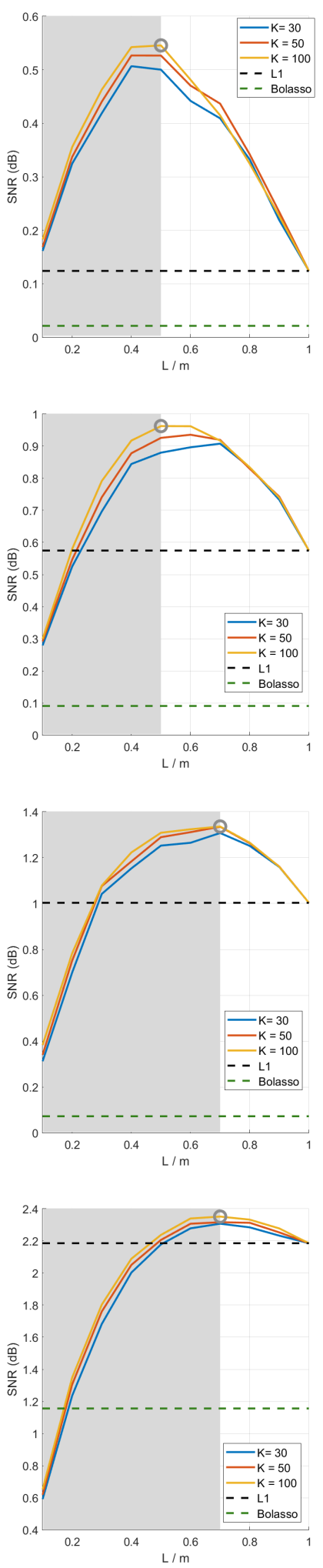

Fig. 5. Performance curves for the subsampling variations of JOBS, Bagging and Bolasso with different $L, K$, and $\ell_{1}$ minimization. SNR $=0$ and the number of measurements $m=50,75,100,150$ from top to bottom. The grey circle highlights the peaks of JOBS, Bagging and Bolasso and the grey area highlights the subsampling ratio at the peak point. JOBS requires smaller $L / m$ than Bagging to achieve peak performance. JOBS and Bagging outperform $\ell_{1}$ minimization and Bolasso when $m$ are small.
JOBS
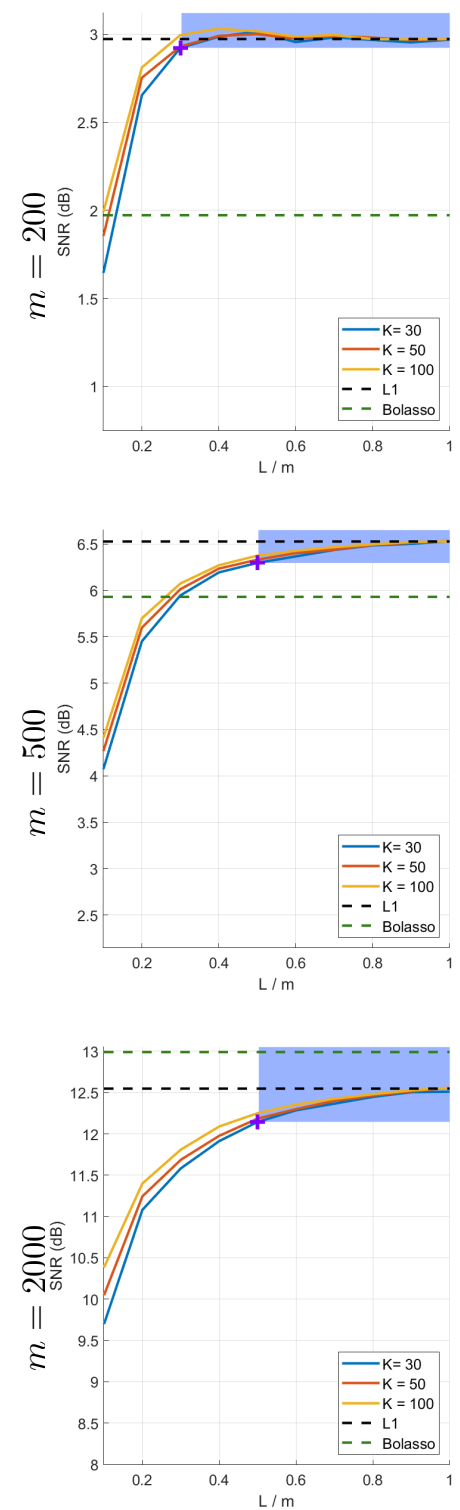

Bagging
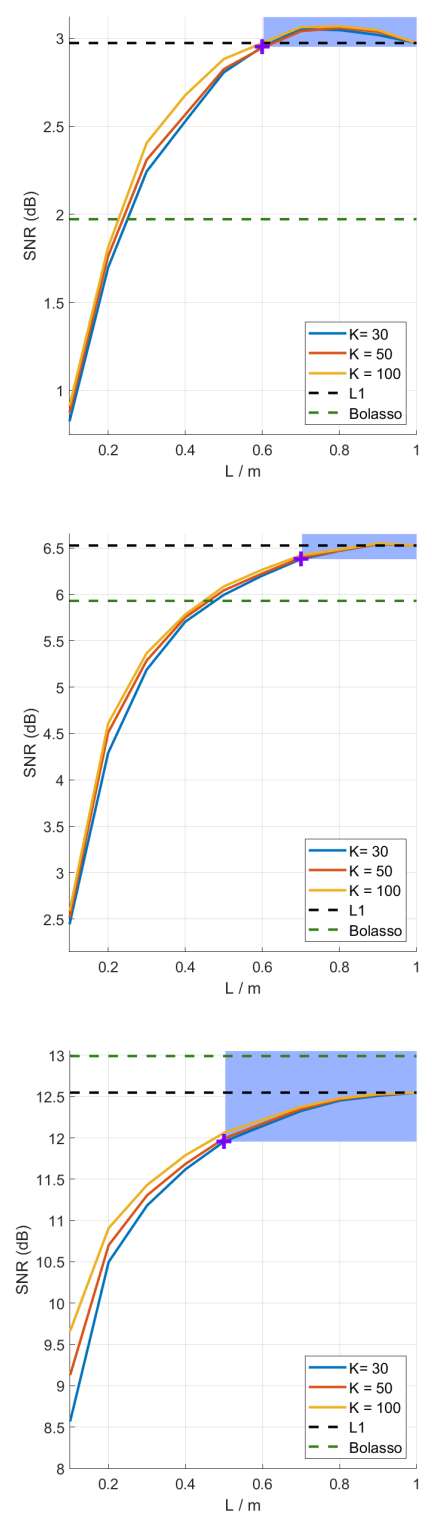

Fig. 6. Performance curves for the subsampling variations of JOBS, Bagging and Bolasso with different $L, K$, and $\ell_{1}$ minimization. SNR $=0 \mathrm{~dB}$ and the number of measurements $m=200,500,2000$ from top to bottom. The purple highlighted area is where at least $95 \%$ of the best performance achieved and within which the minimum $K \times L$ is illustrated by purple cross. JOBS requires smaller $L / m$ than Bagging to achieve acceptable performance.

\section{CONCLUSION AND FUtURE WORK}

We propose and demonstrate JOBS, which is motivated from a powerful bootstrapping idea and improves robustness of sparse recovery in noisy environments through the usage of a collaborative recovery scheme. We analyze BNSP, BRIP for our methods as well as the sample complexity. We further derive error bounds for JOBS and Bagging. The simulations results show that our algorithm consistently outperforms Bagging and Bolasso among most choices of parameters $(L, K)$. JOBS is particularly powerful when the number of measurements $m$ is small. This condition is notoriously difficult, both in terms of improving sparse recovery results and studying the associated theoretical properties. Despite these challenges, JOBS 


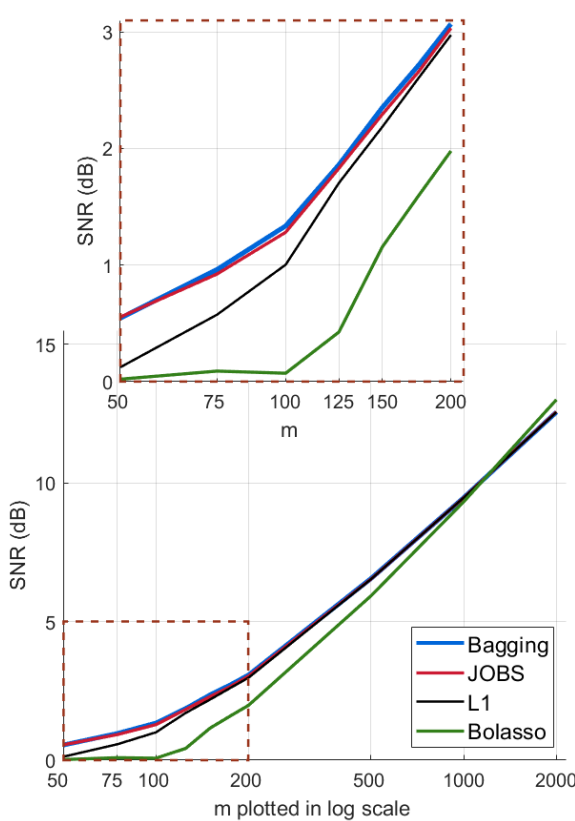

(a) $\mathrm{SNR}=0 \mathrm{~dB}$

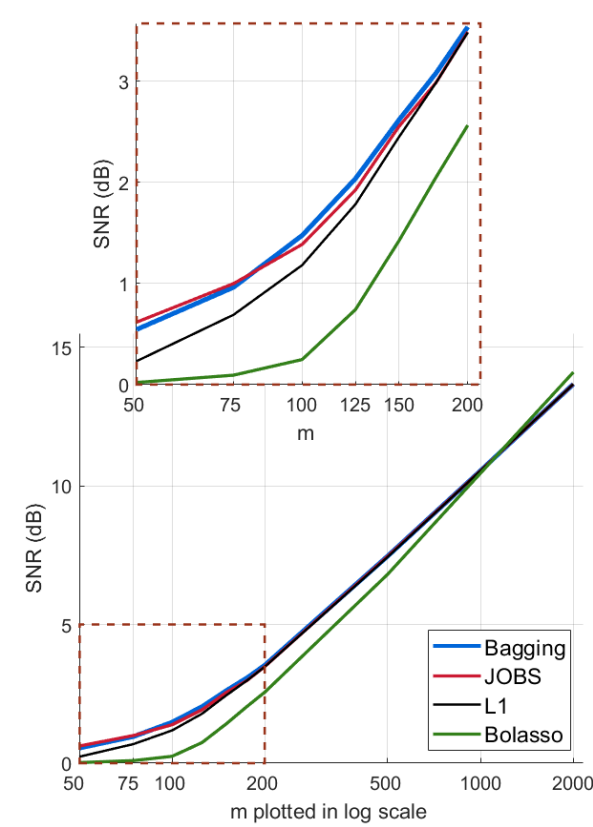

(b) $\mathrm{SNR}=1 \mathrm{~dB}$

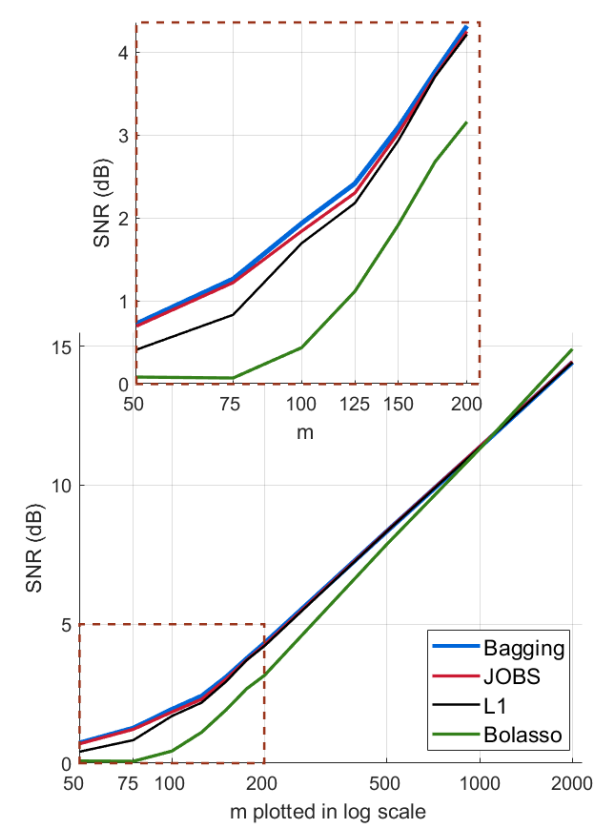

(c) $\mathrm{SNR}=2 \mathrm{~dB}$

Fig. 7. The best performance among all choices of $L$ and $K$ with various number of measurements for subsampling variations of JOBS, Bagging, Bolasso and $\ell_{1}$ minimization. While $m$ is small and lower SNR, the margin between JOBS and $\ell_{1}$ minimization is larger (zoomed-in figures on the top row).

outperforms $\ell_{1}$ minimization by a large margin. JOBS achieves acceptable performance even with very small $L / m$ (around $40 \%$ for the original scheme and $30 \%$ for the subsampling variation) and relative small $K$ (like 30 in our experimental study). The error bounds for JOBS and Bagging show that increasing $K$ will improve the certainty, which is partially validated in the simulation: although it is more computational consuming to choose a larger $K$, increasing $K$ in general gives a better result. Also, for exactly $s$-sparse signals, we have proven that if the RIP condition is satisfied, then for the same $K, L$, JOBS outperforms Bagging. This result matches the large $m$ cases in the simulation, in which the RIP condition should be satisfied. Future work would include applying the algorithm to dictionary learning and classification.

\section{APPENDIX}

\section{A. BNSP for $\ell_{p, 2 \mid \mathcal{B}}$ norm minimization}

Similar to $\ell_{1,2}$ norm in 3 , the definition for $\ell_{p, q}$ norm over block partition $\mathcal{B}$ for vector $\|\boldsymbol{x}\|_{p, q \mid \mathcal{B}}$ is defined as:

$$
\begin{aligned}
\|\boldsymbol{x}\|_{p, q \mid \mathcal{B}}= & \left(\sum_{i=1}^{m}\left\|\boldsymbol{x}\left[\mathcal{B}_{i}\right]^{\boldsymbol{T}}\right\|_{q}^{p}\right)^{1 / p} \\
& =\left\|\left(\left\|\boldsymbol{x}\left[\mathcal{B}_{1}\right]^{\boldsymbol{T}}\right\|_{q}, \ldots,\left\|\boldsymbol{x}\left[\mathcal{B}_{K}\right]^{\boldsymbol{T}}\right\|_{q}\right)\right\|_{p}
\end{aligned}
$$

In the case when $q=2$, its Block Null Space Property is studied. We recall Definition 24 in [29] that gives BNSP for the mixed $\ell_{p, 2}$ norm in the following theorem.

Definition 19 (BNSP for $\ell_{p, 2}$ minimization). For any set $\mathcal{S} \subset\{1,2, \ldots, m\}$ with $\operatorname{card}(\mathcal{S}) \leq s$, a matrix $\boldsymbol{A}$ is said to satisfy the $\ell_{p}, 0<p \leq 1$ block null space property over $\mathcal{B}=\left\{d_{1}, d_{2}, \ldots, d_{m}\right\}$ of order $s$, if

$$
\|\boldsymbol{v}[\mathcal{S}]\|_{p, 2 \mid \mathcal{B}}<\left\|\boldsymbol{v}\left[\mathcal{S}^{c}\right]\right\|_{p, 2 \mid \mathcal{B}},
$$

for all $\boldsymbol{v} \in \operatorname{Null}(\boldsymbol{A}) \backslash\{\mathbf{0}\}$, where $\boldsymbol{v}[\mathcal{S}]$ denotes the vector equal to $\boldsymbol{v}$ on a block index set $\mathcal{S}$ and zero elsewhere.

\section{B. Proof of the reverse direction for noiseless recovery}

Lemma 20. If the $M M V$ problem $\mathbf{P}_{\mathbf{1}}(K), K>1$, has a unique solution, it will be of form $\boldsymbol{X}^{\star}=\left(\boldsymbol{x}^{\star}, \boldsymbol{x}^{\star}, \ldots, \boldsymbol{x}^{\star}\right)$, and then there is a unique solution to $\mathbf{P}_{\mathbf{1}}: \boldsymbol{x}^{\star}$.

Let us proof the other direction. If $\mathbf{P}_{\mathbf{1}}(K)$ has a unique solution, the solution must be in the form of $\boldsymbol{X}^{\star}=\left(\boldsymbol{x}^{\star}, \boldsymbol{x}^{\star}, \ldots, \boldsymbol{x}^{\star}\right)$, and it implies that $\mathbf{P}_{\mathbf{1}}$ has a unique solution $\boldsymbol{x}^{\star}$.

If $\mathbf{P}_{\mathbf{1}}(K)$ has a unique solution, then it is equivalent to say that $\boldsymbol{A}$ satisfied BNSP of order $s$. For all $\boldsymbol{V}=$ $\left(\boldsymbol{v}_{1}, \boldsymbol{v}_{2}, \ldots, \boldsymbol{v}_{k}\right) \neq \boldsymbol{O}, \boldsymbol{v}_{j} \in \operatorname{Null}(\boldsymbol{A})$, we have $\forall \mathcal{S},|\mathcal{S}| \leq$ $s,\|\boldsymbol{V}[\mathcal{S}]\|_{1,2}<\left\|\boldsymbol{V}\left[\mathcal{S}^{c}\right]\right\|_{1,2}$. This implies that $\forall \boldsymbol{V}=$ $(\boldsymbol{v}, \mathbf{0}, \mathbf{0}, \ldots, \mathbf{0}), \boldsymbol{v} \in \operatorname{Null}(\boldsymbol{A}) \backslash\{\mathbf{0}\}$, BNSP is satisfied. Since in this case, except the first column, all other columns are zero and therefore do not contribute to the group norm. Mathematically, for all $\mathcal{S},\|\boldsymbol{V}[\mathcal{S}]\|_{1,2}=\|\boldsymbol{v}[\mathcal{S}]\|_{1}$. We therefore will have the BNSP of order $s$ implies the NSP for $\boldsymbol{A}$ of order $s$.

\section{Proof of Proposition 11}

To proof this proposition, we give alternative form of RIP and BRIP which are stated in the following two propositions. Alternative form of RIP as a function of matrix induced norm is given as the following:

Proposition 21 (Alternative form of RIP). Matrix $\boldsymbol{A}$ has $\ell_{2}$ normalized columns, and $\boldsymbol{A} \in \mathbb{R}^{m \times n}, \mathcal{S} \subset\{1,2, \ldots, n\}$ with size smaller or equal to $s$ and $\boldsymbol{A}_{\mathcal{S}}$ takes columns of $\boldsymbol{A}$ with indices in $\mathcal{S}$. RIP constant of order $s$ of $\boldsymbol{A}, \delta_{s}(\boldsymbol{A})$ is:

$$
\delta_{s}(\boldsymbol{A})=\max _{\mathcal{S} \subseteq\{1,2, \ldots, n\},|\mathcal{S}| \leq s}\left\|\boldsymbol{A}_{\mathcal{S}}^{\boldsymbol{T}} \boldsymbol{A}_{\mathcal{S}}-\boldsymbol{I}\right\|_{2 \rightarrow 2},
$$


where $\boldsymbol{I}$ is an identity matrix of size $s \times s$ and $\|\cdot\|_{2 \rightarrow 2}$ is the induced 2-norm defined as for any matrix $\boldsymbol{A},\|\boldsymbol{A}\|_{2 \rightarrow 2}=$ $\sup _{\boldsymbol{x} \neq \mathbf{0}} \frac{\|\boldsymbol{A} \boldsymbol{x}\|_{2}}{\|\boldsymbol{x}\|_{2}}$.

The proposition 21 can be directly derived from the definition of RIP constant. Similarly, we can derive the alternative form of BRIP constant as a function of matrix induced norm.

Proposition 22 (Alternative form of BRIP). Let matrix $\boldsymbol{A}$ have $\ell_{2}$-normalized columns and let $\mathcal{B}=\left\{\mathcal{B}_{1}, \mathcal{B}_{2}, \ldots, \mathcal{B}_{n}\right\}$ be the group sparsity pattern that defines the row sparsity pattern, with $\mathcal{B}_{i}$ contains all indices corresponding to all elements of the $i-$ th row. $\mathcal{S} \subseteq\{1,2, \ldots, n\}$ and $\mathcal{B}(\mathcal{S})=\left\{\mathcal{B}_{i}, i \in \mathcal{S}\right\}$ be the subsets that takes several groups with group indices in $\mathcal{S}$. and $\boldsymbol{A} \in \mathbb{R}^{m \times n}$ with Block-RIP constant of order $s, \delta_{s \mid \mathcal{B}}(\boldsymbol{A})$ is

$$
\delta_{s \mid \mathcal{B}}=\max _{\mathcal{S} \subseteq\{1,2, \ldots, n\},|\mathcal{S}| \leq s}\left\|\boldsymbol{A}_{\mathcal{B}(\mathcal{S})}^{T} \boldsymbol{A}_{\mathcal{B}(\mathcal{S})}-\boldsymbol{I}\right\|_{2 \rightarrow 2} .
$$

With loss of generality, let us assume that all columns of $\boldsymbol{A}$ in the original $\ell_{1}$ minimization have unit $\ell_{2}$ norms. As a consequence, sub-matrices of $\boldsymbol{A}: \boldsymbol{A}\left[\mathcal{I}_{j}\right] \mathrm{s}$ in general will not guaranteed to have normalized columns. Then, we normalize the columns of these matrices using the following normalization procedure. For $M \in \mathbb{R}^{L \times n}$, a matrix that does not have any zero column, $\boldsymbol{Q}(\boldsymbol{M}) \in \mathbb{R}^{n \times n}$ is a normalization matrix of $\boldsymbol{M}$ such that $\boldsymbol{M Q}(\boldsymbol{M})$ has $\ell_{2}$-normalized columns. Clearly, the normalization matrix of $M$ can be obtained:

$$
\boldsymbol{Q}(\boldsymbol{M})=\operatorname{diag}\left(\left\|\boldsymbol{m}_{1}\right\|_{2}^{-1},\left\|\boldsymbol{m}_{2}\right\|_{2}^{-1}, \ldots,\left\|\boldsymbol{m}_{n}\right\|_{2}^{-1}\right),
$$

where $\boldsymbol{m}_{j}$ denotes $j$-th column of $\boldsymbol{M}$.

Similary, construct $\boldsymbol{Q}_{j}$ s using 66 to normalize $\boldsymbol{A}\left[\mathcal{I}_{j}\right] \mathrm{s}$. Here, $\boldsymbol{A}\left[\mathcal{I}_{j}\right]$ s are sub-rows of $\boldsymbol{A}$, the norm of all columns in $\boldsymbol{A}\left[\mathcal{I}_{j}\right]$ are all less than those of $\boldsymbol{A}$ (which are all equal to 1 ). Since $\boldsymbol{Q}_{j}$ contains reciprocals of norm of columns of matrix, $\boldsymbol{Q}_{j}$ s are diagonal matrices with their diagonals greater or equal to 1 .

Let $\boldsymbol{A}^{J}=$ block_diag $\left(\boldsymbol{A}\left[\mathcal{I}_{1}\right], \boldsymbol{A}\left[\mathcal{I}_{2}\right], \ldots, \boldsymbol{A}\left[\mathcal{I}_{K}\right]\right)$ and $\boldsymbol{Q}^{J}=$ block_diag $\left(\boldsymbol{Q}_{1}, \boldsymbol{Q}_{2}, \ldots, \boldsymbol{Q}_{K}\right)$. Then columns of $\boldsymbol{A}^{J} \boldsymbol{Q}^{J}=$ block_diag $\left(\boldsymbol{A}\left[\mathcal{I}_{1}\right] \boldsymbol{Q}_{1}, \boldsymbol{A}\left[\mathcal{I}_{2}\right] \boldsymbol{Q}_{2}, \ldots, \boldsymbol{A}\left[\mathcal{I}_{K}\right] \boldsymbol{Q}_{K}\right)$ all have unit norms. We consider the BRIP constant for $A^{J}$. In this derivation, column selection of a matrix is written as a right multiplication of matrix $\boldsymbol{I}_{\mathcal{S}}(\cdot)$.

$$
\begin{gathered}
\delta_{s \mid \mathcal{B}}\left(\boldsymbol{A}^{\boldsymbol{J}}\right) \\
=\max _{\mathcal{S} \subseteq\{1,2, . ., n\},|\mathcal{S}| \leq s}\left\|\left(\boldsymbol{A}^{\boldsymbol{J}} \boldsymbol{Q}^{\boldsymbol{J}} \boldsymbol{I}_{\mathcal{B}(\mathcal{S})}\right)^{\boldsymbol{T}} \boldsymbol{A}^{\boldsymbol{J}} \boldsymbol{Q}^{\boldsymbol{J}} \boldsymbol{I}_{\mathcal{B}(\mathcal{S})}-\boldsymbol{I}\right\|_{2 \rightarrow 2} \\
=\max _{\substack{\mathcal{S} \subseteq\{1,2, . ., n\} \\
|\mathcal{S}| \leq s}} \max _{j}\left\|\left(\boldsymbol{A}\left[\mathcal{I}_{j}\right] \boldsymbol{Q}_{j} \boldsymbol{I}_{\mathcal{S}}\right)^{\boldsymbol{T}} \boldsymbol{A}\left[\mathcal{I}_{j}\right] \boldsymbol{Q}_{j} \boldsymbol{I}_{\mathcal{S}}-\boldsymbol{I}\right\|_{2 \rightarrow 2} \\
=\max _{\substack{\mathcal{S} \subseteq\{1,2, . ., n\} \\
|\mathcal{S}| \leq s}} \| \text { block_diag }\left(\left(\boldsymbol{A}\left[\mathcal{I}_{1}\right] \boldsymbol{Q}_{1} \boldsymbol{I}_{\mathcal{S}}\right)^{\boldsymbol{T}} \boldsymbol{A}\left[\mathcal{I}_{1}\right] \boldsymbol{Q}_{1} \boldsymbol{I}_{\mathcal{S}}-\boldsymbol{I},\right. \\
\left.\quad \ldots,\left(\boldsymbol{A}\left[\mathcal{I}_{K}\right] \boldsymbol{Q}_{K} \boldsymbol{I}_{\mathcal{S}}\right)^{\boldsymbol{T}} \boldsymbol{A}\left[\mathcal{I}_{K}\right] \boldsymbol{Q}_{K} \boldsymbol{I}_{\mathcal{S}}-\boldsymbol{I}\right) \|_{2 \rightarrow 2}
\end{gathered}
$$

The induced 2-norm of a matrix equals to the max singular value of $\|\boldsymbol{D}\|_{2 \rightarrow 2}=\sigma_{\max }(\boldsymbol{D})$ and if $\boldsymbol{D}$ is a block diagonal ma- $\operatorname{trix} \boldsymbol{D}=\operatorname{diag}\left(\boldsymbol{D}_{1}, \boldsymbol{D}_{2}, \ldots, \boldsymbol{D}_{n}\right), \sigma_{\max }(\boldsymbol{D})=\max \sigma_{\max }\left(\boldsymbol{D}_{j}\right)$. We use this property here. Then

$$
\begin{aligned}
& \delta_{s \mid \mathcal{B}}\left(\boldsymbol{A}^{\boldsymbol{J}}\right) \\
& =\max _{\substack{\mathcal{S} \subseteq\{1,2, . ., n\} \\
|\mathcal{S}| \leq s}} \max _{j}\left\|\left(\boldsymbol{A}\left[\mathcal{I}_{j}\right] \boldsymbol{Q}_{j} \boldsymbol{I}_{\mathcal{S}}\right)^{\boldsymbol{T}} \boldsymbol{A}\left[\mathcal{I}_{j}\right] \boldsymbol{Q}_{j} \boldsymbol{I}_{\mathcal{S}}-\boldsymbol{I}\right\|_{2 \rightarrow 2} \\
& =\max _{j} \max _{\substack{\mathcal{S} \subseteq\{1,2, \ldots, n\} \\
|\mathcal{S}| \leq s}}\left\|\left(\boldsymbol{A}\left[\mathcal{I}_{j}\right] \boldsymbol{Q}_{j} \boldsymbol{I}_{\mathcal{S}}\right)^{\boldsymbol{T}} \boldsymbol{A}\left[\mathcal{I}_{j}\right] \boldsymbol{Q}_{j} \boldsymbol{I}_{\mathcal{S}}-\boldsymbol{I}\right\|_{2 \rightarrow 2} \\
& =\max _{j} \delta_{s}\left(\boldsymbol{A}\left[\mathcal{I}_{j}\right]\right) \text {. }
\end{aligned}
$$

\section{The Close Form of Birthday Problem}

We generate $L$ samples from $m$ samples uniformly at random with replacement $(L \leq m)$. Let $V$ denote the number of distinct samples among $L$ samples. Clearly we have $V \in[1, L]$ and the probability mass function is given by [36], same as the famous Birthday problem in statistics:

$$
\begin{aligned}
& \mathbb{P}(V=v)=\left(\begin{array}{c}
m \\
v
\end{array}\right) \sum_{j=0}^{v}(-1)^{j}\left(\begin{array}{l}
v \\
j
\end{array}\right)\left(\frac{v-j}{m}\right)^{L}, \\
& v=1,2, \ldots, L
\end{aligned}
$$

In our problem, we are interested in finding the lower bound of $V$ with certainty level $1-\alpha$

$\mathbb{P}(V \geq d)=\sum_{v=d}^{L}\left(\begin{array}{l}m \\ v\end{array}\right) \sum_{j=0}^{v}(-1)^{j}\left(\begin{array}{l}v \\ j\end{array}\right)\left(\frac{v-j}{m}\right)^{L} \geq 1-\alpha$.

Therefore for

$$
1 \geq \alpha \geq \sum_{v=0}^{d-1}\left(\begin{array}{c}
m \\
v
\end{array}\right) \sum_{j=0}^{v}(-1)^{j}\left(\begin{array}{l}
v \\
j
\end{array}\right)\left(\frac{v-j}{m}\right)^{L},
$$

equation 68 is satisfied.

\section{E. Proof of Lemma 18}

To prove of this lemma, we would need Markovs' inequality for non-negative random variables: let $X$ be a non-negative random variable and suppose that $\mathbb{E} X$ exists, for any $t>0$, we have:

$$
\mathbb{P}\{X>t\} \leq \frac{\mathbb{E} X}{t} .
$$

We also need the upper bound of the moment generating function (MGF) of random variable $Y$ : suppose that $a \leq Y \leq b$, then for all $t \in \mathbb{R}$ :

$$
\mathbb{E} \exp \{t Y\} \leq \exp \left\{t \mathbb{E} Y+\frac{t^{2}(b-a)^{2}}{8}\right\} .
$$

Then we start prove the Lemma 18 , for $t>0$.

$$
\begin{aligned}
& \mathbb{P}\left\{\sum_{i=1}^{n} Y_{i} \geq n \epsilon\right\}=\mathbb{P}\left\{\exp \left\{\sum_{i=1}^{n} Y_{i}\right\} \geq \exp \{n \epsilon\}\right\} \\
& \quad=\mathbb{P}\left\{\exp \left\{t \sum_{i=1}^{n} Y_{i}\right\} \geq \exp \{t n \epsilon\}\right\}
\end{aligned}
$$


using the Markov inequality in (70),

$$
\begin{aligned}
& \leq \exp \{-t n \epsilon\} \mathbb{E}\left\{\exp \left\{t \sum_{i=1}^{n} Y_{i}\right\}\right\} \\
& =\exp \{-t n \epsilon\} \mathbb{E}\left\{\Pi_{i=1}^{n} \exp \left\{t Y_{i}\right\}\right\} \\
& =\exp \{-t n \epsilon\} \Pi_{i=1}^{n} \mathbb{E}\left\{\exp \left\{t Y_{i}\right\}\right\},
\end{aligned}
$$

and by upper bound for MGF in (71)

$$
\begin{aligned}
& \leq \exp \{-t n \epsilon\}\left(\exp \left\{t \mathbb{E} Y+\frac{t^{2}(b-a)^{2}}{8}\right\}\right)^{n} \\
& =\exp \left\{-t n \epsilon+t n \mathbb{E} Y+\frac{t^{2}(b-a)^{2} n}{8}\right\} .
\end{aligned}
$$

The right hand side is a convex function respect to $t$, taking the derivative with respect to $t$ and set it zero, we obtain the optimal $t, t^{\star}=\frac{4 \epsilon-4 E Y}{(b-a)^{2}}$ and the right hand side is minimized:

$\exp \left\{-t^{\star} n \epsilon+t^{\star} n \mathbb{E} Y+\frac{t^{\star 2}(b-a)^{2} n}{8}\right\}=\exp \left\{\frac{-2 n(\epsilon-\mathbb{E} Y)^{2}}{(b-a)^{2}}\right\}$.

\section{REFERENCES}

[1] Balas Kausik Natarajan. Sparse approximate solutions to linear systems. SIAM J. on computing, 24(2):227-234, 1995.

[2] Scott Shaobing Chen, David L Donoho, and Michael A Saunders. Atomic decomposition by basis pursuit. SIAM review, 43(1):129-159, 2001.

[3] Robert Tibshirani. Regression shrinkage and selection via the lasso. $J$. of the Royal Stat. Society. Series B, pages 267-288, 1996.

[4] Emmanuel J Candes. The restricted isometry property and its implications for compressed sensing. Comptes Rendus Mathematique, 346(9):589-592, 2008.

[5] Emmanuel J Candes, Justin Romberg, and Terence Tao. Robust uncertainty principles: Exact signal reconstruction from highly incomplete frequency information. IEEE Trans. on info. theory, 52(2):489-509, 2006.

[6] David L Donoho. Compressed sensing. IEEE Trans. on info. theory, 52(4):1289-1306, 2006.

[7] Emmanuel Candess and Justin Romberg. Sparsity and incoherence in compressive sampling. Inverse prob., 23(3):969, 2007.

[8] Michal Aharon, Michael Elad, and Alfred Bruckstein. K-SVD: An algorithm for designing overcomplete dictionaries for sparse representation. Sig. Proc., IEEE Trans. on, 54(11):4311-4322, 2006.

[9] Jianchao Yang, John Wright, Thomas S Huang, and Yi Ma. Image super-resolution via sparse representation. IEEE trans. on image proc., 19(11):2861-2873, 2010.

[10] Luoluo Liu, Trac D Tran, and Sang Peter Chin. Partial face recognition: A sparse representation-based approach. In 2016 IEEE Int. Conf. on Acoustics, Speech and Signal Processing, ICASSP 2016, Shanghai, China March 20-25, 2016, pages 2389-2393, 2016.

[11] Yi Chen, Thong T Do, and Trac D Tran. Robust face recognition using locally adaptive sparse representation. In Image Proc. (ICIP), 2010 17th IEEE Int. Conf. on, pages 1657-1660. IEEE, 2010.

[12] Alex Krizhevsky, Ilya Sutskever, and Geoffrey E Hinton. Imagenet classification with deep convolutional neural networks. In Advances in neural information processing systems, pages 1097-1105, 2012.

[13] Bradley Efron. Bootstrap methods: another look at the jackknife. The Annals of Stat., 7(1):1-26, 1979.

[14] Leo Breiman. Bagging predictors. Machine learning, 24(2):123-140, 1996.

[15] Francis R Bach. Bolasso: model consistent lasso estimation through the bootstrap. In Proceedings of the 25th int. conf. on Machine learning, pages 33-40. ACM, 2008.

[16] Jie Chen and Xiaoming Huo. Theoretical results on sparse representations of multiple-measurement vectors. IEEE Trans. on Sig. Proc., 54(12):4634 4643, 2006.

[17] Yonina C Eldar and Moshe Mishali. Robust recovery of signals from a structured union of subspaces. Info. Theory, IEEE Trans. on, 55(11):53025316,2009

[18] Joel A Tropp. Algorithms for simultaneous sparse approximation. part ii: Convex relaxation. Sig. Proc., 86(3):589-602, 2006.
[19] Shane F Cotter, Bhaskar D Rao, Kjersti Engan, and Kenneth KreutzDelgado. Sparse solutions to linear inverse problems with multiple measurement vectors. IEEE Trans. on Sig. Proc., 53(7):2477-2488, 2005.

[20] E. van den Berg and M. P. Friedlander. Probing the pareto frontier for basis pursuit solutions. SIAM J. on Scientific Computing, 31(2):890-912, 2008.

[21] Stephen Boyd, Neal Parikh, Eric Chu, Borja Peleato, and Jonathan Eckstein. Distributed optimization and statistical learning via the alternating direction method of multipliers. Foundations and Trends in Machine Learning, 3(1):1-122, 2011.

[22] Dror Baron, Marco F Duarte, Michael B Wakin, Shriram Sarvotham, and Richard G Baraniuk. Distributed compressive sensing. arXiv preprint arXiv:0901.3403, 2009.

[23] Reinhard Heckel and Helmut Bolcskei. Joint sparsity with different measurement matrices. In Communication, Control, and Computing (Allerton), 2012 50th Annual Allerton Conf. on, pages 698-702. IEEE, 2012.

[24] Liang Sun, Jun Liu, Jianhui Chen, and Jieping Ye. Efficient recovery of jointly sparse vectors. In Advances in Neural Info. Proc. Systems, pages 1812-1820, 2009.

[25] Francis R Bach. Consistency of the group lasso and multiple kernel learning. The J. of Machine Learning Research, 9:1179-1225, 2008

[26] Stephen J Wright, Robert D Nowak, and Mario AT Figueiredo. Sparse reconstruction by separable approximation. IEEE Trans. on Sig. Proc., 57(7):2479-2493, 2009

[27] J. Liu, S. Ji, and J. Ye. SLEP: Sparse Learning with Efficient Projections. Arizona State University, 2009

[28] Wei Deng, Wotao Yin, and Yin Zhang. Group sparse optimization by alternating direction method. In Rice CAAM Report TR11-06, pages 88580R-88580R. International Society for Optics and Photonics, 2011.

[29] Yi Gao, Jigen Peng, and Yuan Zhao. On the null space property of $\ell_{q}$-minimization for in compressed sensing. 2015, 2015.

[30] Yonina C Eldar and Moshe Mishali. Block sparsity and sampling over a union of subspaces. In Digital Signal Processing, 2009 16th International Conference on, pages 1-8. IEEE, 2009.

[31] Albert Cohen, Wolfgang Dahmen, and Ronald DeVore. Compressed sensing and best $k$-term approximation. Journal of the American mathematical society, 22(1):211-231, 2009.

[32] Richard Baraniuk, Mark Davenport, Ronald DeVore, and Michael Wakin A simple proof of the restricted isometry property for random matrices. Constructive Approx., 28(3):253-263, 2008.

[33] Wassily Hoeffding. Probability inequalities for sums of bounded random variables. J. of the American statistical association, 58(301):13-30, 1963.

[34] Peter Lukas Bühlmann and Bin Yu. Explaining bagging. In Research report/Seminar für Statistik, Eidgenössische Technische Hochschule Zürich, volume 92. Seminar für Statistik, Eidgenössische Technische Hochschule (ETH), 2000.

[35] Peter Buhlmann. Bagging, subagging and bragging for improving some prediction algorithms. In Research Report. Seminar Fur Statistik, ETH Zurich, Switzerland, 2003.

[36] The birthday problem. http://www.math.uah.edu/stat/urn/Birthday.html Creative Commons License. 